typeset using JPSJ.sty $<$ ver. $0.7 f>$

\title{
Magnetic and Orbital States and Their Phase Transition of the Perovskite-Type Ti Oxides: Strong Coupling Approach
}

\author{
Masahito Mochizuki and Masatoshi Imada \\ Institute for Solid State Physics, University of Tokyo, \\ 5-1-5 Kashiwa-no-ha, Kashiwa, Chiba 277-8581
}

(Received October 28, 2018)

\begin{abstract}
The properties and mechanism of the magnetic phase transition of the perovskite-type $\mathrm{Ti}$ oxides, which is driven by the Ti-O-Ti bond angle distortion, are studied theoretically by using the effective spin and pseudospin Hamiltonian with strong Coulomb repulsion. It is shown that the A-type antiferromagnetic (AFM(A)) to ferromagnetic (FM) phase transition occurs as the $\mathrm{Ti}-\mathrm{O}-\mathrm{Ti}$ bond angle is decreased. Through this phase transition, the orbital state changes only little whereas the spin-exchange coupling along the $c$-axis is expected to change from positive to negative nearly continuously and approaches zero at the phase boundary. The resultant strong two-dimensionality in the spin coupling causes rapid suppression of the critical temperature, as observed experimentally. It may induce large quantum fluctuations in this region.
\end{abstract}

KEYWORDS: perovskite-type $\mathrm{Ti}$ oxides, $\mathrm{GdFeO}_{3}$-type distortion, $d$-type Jahn-Teller distortion, orbital degrees of freedom, orbital ordering, second-order perturbation theory, A-type antiferromagnetism, twodimensional spin coupling, Mermin and Wagner's theorem

\section{§1. Introduction}

Electronic and magnetic properties of perovskite-type transition-metal oxides with strong Coulomb correlations have recently attracted considerable interest from the viewpoint of a complex interplay of charge, spin and orbital degrees of freedom. The chemical formula of these compounds is $R M \mathrm{O}_{3}$, where $R$ denotes a trivalent rareearth ion (i.e., La, $\mathrm{Pr}, \mathrm{Nd}, \ldots, \mathrm{Y}$ ) and $M$ is a transitionmetal ion (i.e., $\mathrm{Ti}, \mathrm{V}, \ldots, \mathrm{Ni}, \mathrm{Cu}$ ). This system is appropriate for a systematic study on the roles of orbital degrees of freedom since we can control a certain kind of lattice parameters as magnitudes of some structural distortions, which strongly affect the one-electron bandwidth, the lifting of the orbital-level degeneracy and the ways of orbital hybridization.

Theoretically, several single-band models have succeeded in explaining several properties. However, within single-band models, it is hard to explain such phenomena as magnetic orderings accompanied by orbital orderings. According to the pioneering work of Kugel and Khomskii, it is important to take account of the $3 d$-level degeneracy when we consider the magnetic and electronic properties of these compounds ${ }^{1-4)}$. They have also pointed out that the magnetic ordering and the orbital ordering are closely related. Usually, $\mathrm{MO}_{6}$ octahedron in perovskite structure undergoes some lattice distortions which work as lowering of the symmetry and lifting of the level degeneracy. Consequently, the structure yields more complicated magnetic properties of these compounds.

Perovskite-type Ti oxides $R \mathrm{TiO}_{3}$ ( $R$ being a trivalent rare-earth ion) is a typical Mott-Hubbard insulator ${ }^{5)}$. $\mathrm{Ti}^{3+}$ has a $3 d^{1}$ configuration, and one of the threefold $t_{2 g}$ orbitals is occupied at each transition-metal site $\left(t_{2 g}^{1}\right.$ configuration). They have also attracted interest since

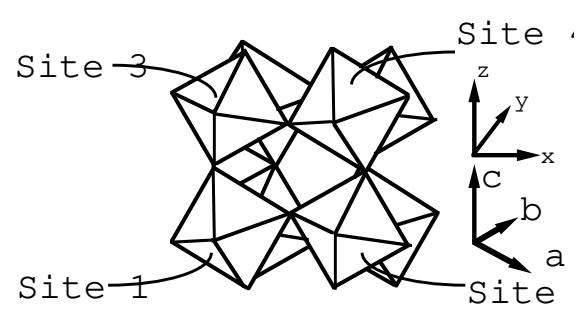

Fig. 1. $\mathrm{GdFeO}_{3}$-type distortion.

these systems show various magnetic and orbital ordered phases. Moreover, it is required to take the spin and orbital degrees of freedom into consideration on an equal footing to explain such rich phases.

The crystal structure is an orthorhombically distorted perovskite $\left(\mathrm{GdFeO}_{3}\right.$-type distortion) in which the $\mathrm{TiO}_{6}$ octahedra forming the perovskite lattice tilt alternatingly. In this distortion, the unit cell contains four octahedra, as shown in Fig. 1. The magnitude of the distortion (in other words, degree of the Ti-O-Ti bond-angle distortion) depends on the ionic radii of the $R$ ion. With a small ionic radius of the $R$ ion, the lattice structure is more distorted and the bond angle is decreased more significantly from $180^{\circ}$. For example, in $\mathrm{LaTiO}_{3}$, the bond angle is $157^{\circ}$ ( $a b$-plane) and $156^{\circ}$ (c-axis), but $144^{\circ}$ ( $a b$ plane) and $140^{\circ}$ (c-axis) in $\mathrm{YTiO}_{3}{ }^{6}$. We can control the magnitude or the Ti-O-Ti bond angle by the use of solid-solution systems $\mathrm{La}_{1-y} \mathrm{Y}_{y} \mathrm{TiO}_{3}$ or in $R \mathrm{TiO}_{3}$, by varying the $R$ ions from $\mathrm{La}, \mathrm{Pr}, \mathrm{Nd}, \ldots$, to $\mathrm{Y}$. In particular, by varying the $\mathrm{Y}$ concentration in $\mathrm{La}_{1-y} \mathrm{Y}_{y} \mathrm{TiO}_{3}$, we can control the bond angle almost continuously from $156^{\circ}(y=0)$ to $140^{\circ}(y=1)$. Mainly, the distortion has two kind of roles. One is the "band-width control". Since the transfers of electrons on Ti $3 d$ orbitals are gov- 

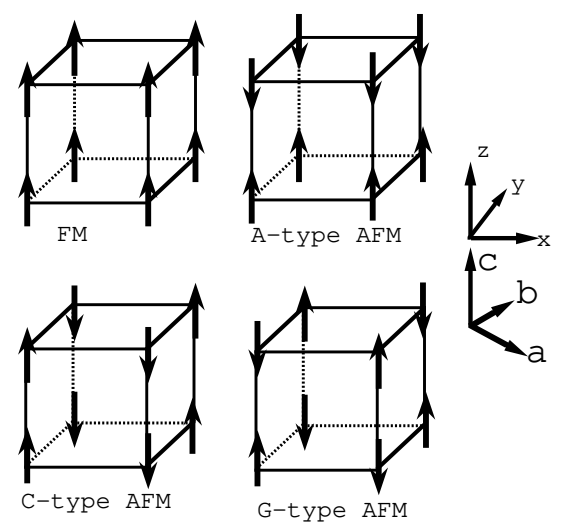

Fig. 2. Typical magnetic structures for the perovskites

erned by the super-transfer processes mediated by the $\mathrm{O}$ $2 p$ states in perovskite-type transition-metal oxides, the Ti-O-Ti bond angle distortion causes the reduction of $3 d$ $t_{2 g}$-electron bandwidth $W$ critically. Another role of the Ti-O-Ti bond angle distortion is a symmetry relaxation of the indirect $d-d$ transfer between neighboring $3 d$ orbitals with different symmetries. In the cubic-perovskite lattice with no distortion, an electron in an $t_{2 g}$ orbital with a certain symmetry can transfer to that with the same symmetry at the neighboring site. On the other hand, the $\mathrm{GdFeO}_{3}$-type distortion relaxes the symmetry restriction and makes it possible for an electron in a $t_{2 g}$ orbital with one symmetry to transfer to that with another symmetry at the neighboring site. In addition, the indirect transfers between neighboring $t_{2 g}$ and $e_{g}$ orbitals are increased critically as the distortion increases. This is one of the reasons for a rich magnetic phase diagram as a function of the bond angle since the magnetic and orbital orderings are strongly associated with the way of orbitalhybridization as was studied previously by Kanamori ${ }^{7,8)}$, Goodenough $^{9,10)}$, Kugel and Khomskii ${ }^{1-4)}$. In this paper, $\mathrm{GdFeO}_{3}$-type distortion is simulated by rotating the $\mathrm{TiO}_{6}$ octahedra by angle $+\theta$ and $-\theta$ about the $(1,1,1)$ and $(-1,-1,1)$ axes with respect to the $x, y$, and $z$ axes.

In $\mathrm{YTiO}_{3}$, a $d$-type Jahn-Teller (JT) distortion has been observed in which the longer and shorter Ti-O bond lengths are $\sim 2.08 \AA$ and $\sim 2.02 \AA$, respectively ${ }^{11)}$. On the other hand, $\mathrm{LaTiO}_{3}$ exhibits a small or no JT distortion. In the $d$-type JT distortion, the elongated axes of the octahedra are parallel along the $c$-axis. In the JT distortion, antibonding character between Ti $3 d$ and $\mathrm{O}$ $2 p$ orbitals is reduced and the orbitals directed in the elongated direction are lowered in energy. For example, if the $x$-axis is elongated, among the threefold $t_{2 g}$ orbitals, the $x y$ and $z x$ are lowered relative to the $y z$. As a result, in the $d$-type JT distortion the $x y$ and $y z$ orbitals are stabilized at sites 1 and 3 and the $x y$ and $z x$ orbitals are stabilized at sites 2 and 4 . The JT distortion plays an important role on orbital and magnetic orderings.

Recently, electronic and magnetic phase diagrams have been investigated intensively as functions of the magnitude of a Ti-O-Ti bond angle distortion ${ }^{12-15)}$. In the less-distorted or in a La-rich $(y<0.6)$ region, the system shows an AFM ground state. In particular, $\mathrm{LaTiO}_{3}(y=$
0.0) shows a G-type $\operatorname{AFM}(\operatorname{AFM}(G))$ ground state with a magnetic moment of $0.45 \mu_{\mathrm{B}}$, in which the spins are aligned antiferromagnetically in all directions ${ }^{16)}$. With increasing $\mathrm{Y}$ concentration or varying the $R$ site with smaller-sized ions (a decrease of the Ti-O-Ti bond angle), the Nèel temperature $\left(T_{N}\right)$ decreases rapidly and is suppressed to almost zero, subsequently a FM ordering appears. This rapid decrease of $T_{N}$ is not well explained by the conventional models. Its origin is an issue of interest. In the relatively distorted or Y-rich region, the system has a FM ground state. In $\mathrm{YTiO}_{3}(y=1.0)$, the value of the magnetic moment is $0.84 \mu_{\mathrm{B}}$ and the Curie temparature $\left(T_{C}\right)$ takes $\sim 30 \mathrm{~K}^{17)}$. This ferromagnetism is hardly explained by a simple single-band Hubbard model and requires consideration of the $d$-level degeneracy.

Strong suppressions of $T_{N}$ and $T_{C}$ around the AFMFM transition point imply a continuous-type transition at $T=0$. At first sight, this is a puzzling feature, because we expect the first-order transition between completely different symmetry breaking at $T=0$ and $T_{N}$ and $T_{C}$ may remain nonzero at the transition point. Clarifying the mechanism of this continuous-type transition is the purpose of our study in this paper.

Recent model Hartree-Fock(HF) studies based on a multiband $d-p$ model have succeeded in reproducing the spin structures of both end compounds, $\mathrm{LaTiO}_{3}$ and $\mathrm{YTiO}_{3}{ }^{18,19)}$. According to the studies, the $t_{2 g}^{1}$ configuration in $\mathrm{LaTiO}_{3}$ with smaller $\mathrm{GdFeO}_{3}$-type distortion and with small or no JT distortion is well described by the spin-orbit ground state, out of which two states with antiparallel orbital and spin moments, $\frac{1}{\sqrt{2}}\left(z^{\prime} x^{\prime}+i y^{\prime} z^{\prime}\right) \uparrow$ and $\frac{1}{\sqrt{2}}\left(z^{\prime} x^{\prime}-i y^{\prime} z^{\prime}\right) \downarrow$, with the $z^{\prime}$-axis pointing in the $(1,1,1)$-direction in terms of the $x, y$ and $z$ axes are alternating between nearest neighbors, favored both by the spin-orbit interaction and by the super-exchange interaction. As a result, the $\operatorname{AFM}(\mathrm{G})$ state in which the spins point in the $z^{\prime}$-direction is expected to be realized. In addition, the total energies of various spin and orbital structures are calculated as functions of the Ti$\mathrm{O}$-Ti bond angle in the large $d$-type JT distortion. As a result, in the large $\mathrm{GdFeO}_{3}$-type distortion, a FM solution accompanied by an orbital ordering was proved to be stabilized. Moreover, the band calculation for $\mathrm{YTiO}_{3}$ by the generalized gradient approximation (GGA) and the local spin-density-approximation (LSDA) in which the $\mathrm{GdFeO}_{3}$-type distortion is taken into account also succeeded in reproducing a FM spin state with an orbital ordering in $\mathrm{YTiO}_{3}{ }^{20)}$.

In these weak coupling approach, however, the $d$ - $d$ Coulomb interaction is treated in an approximate and averaged way while in perovskite-type $\mathrm{Mn}$ oxides, the importance of the interaction in the degenerate orbitals has been pointed out in terms of the magnetic and orbital ordering by Kanamori ${ }^{7,8)}$, Goodenough ${ }^{9,10)}$, Kugel and Khomskii ${ }^{1-4)}$. Actually, the intrasite Coulomb interaction is also much larger than the other leading energies of the parameters in Ti systems. The GGA and LSDA are also not sufficient for the description of the strongly localized electron states. It is well-known that 
the GGA and LSDA have a tendency to underestimate the magnitude of the band gaps. Although the FM state is obtained both in LSDA and GGA for $\mathrm{YTiO}_{3}$, that obtained in LSDA is metallic and that obtained in GGA is half-metallic, being in disagreement with the experimental result that $\mathrm{YTiO}_{3}$ is insulating with optical gap of $1.0 \mathrm{eV}^{14)}$. Moreover, any magnetic solutions cannot be obtained for $\mathrm{LaTiO}_{3}$ in LSDA. These facts indicate that the electron correlation should be treated in more sophisticated way when we consider the electronic properties of these compounds.

Moreover, the magnetic and orbital states realized in the actual systems and the properties of their phase transitions are still controversial. If the JT distortion is small, the spin-orbit interaction is substantial for the electronic states of the $\mathrm{Ti} 3 \mathrm{~d}$ electrons and hence, the system is well described by the spin-orbit ground state. On the other hand, in the region of large JT distortion, the energy splitting due to the JT distortion becomes comparable or larger than the spin-orbit level-splitting. The situation is no longer the same as that in the region of small JT distortion. The AFM phase realized in such a large JT distortion can be qualitatively different from that realized in $\mathrm{LaTiO}_{3}$. Thus the magnetic and orbital states realized in the moderately distorted region between $\mathrm{LaTiO}_{3}$ and $\mathrm{YTiO}_{3}$ are issues of interest. In addition, the nature of the magnetic phase transition, which is considered to occur in the large JT-distortionregion remains insufficiently clarified.

In this paper, we study the magnetic and the orbital orderings and their phase transitions in perovskite-type $\mathrm{Ti}$ oxides as functions of a Ti-O-Ti bond-angle distortion by using an effective spin and pseudospin Hamiltonian constructed through the second-order perturbational expansion with respect to the transfer terms in the limit of the strong Coulomb repulsion. In this Hamiltonian, the full degeneracy of $\mathrm{Ti} 3 d$ orbitals and on-site Coulomb and exchange interactions are taken into account. In addition, effects of $\mathrm{GdFeO}_{3}$-type and JT distortions are also considered by modifications of the hopping integrals and splitting of the $t_{2 g}$ and $e_{g}$ levels. Our approach is appropriate for the systematic study on the properties and mechanism of the magnetic and orbital phase transitions since the origin of the stabilization of magnetic and orbital ordered states are attributed to the second-order perturbational energy gains with respect to the indirect $d-d$ transfers and, moreover, their phase transition are caused by the competition of their energy gains. These energy gains are easily investigated in our approach by estimating the anisotropic transfer-amplitudes and the level-splitting energies which are driven by the lattice structure and several lattice distortions.

We show that:

- The $e_{g}$ orbital degrees of freedom play important roles on the magnetic phase transition in this system;

- as the $\mathrm{GdFeO}_{3}$-type distortion increases, the spinexchange interaction along the $c$-axis changes from positive to negative due to the super-exchange processes mediated by the $e_{g}$ orbitals, but the orbital state does not change;
- the AFM-FM phase transition point is well described by the two-dimensional Heisenberg model so that $T_{N}$ and $T_{C}$ are suppressed to almost zero;

- large quantum fluctuations and anisotropy in the spin-wave dispersion are expected to be observed.

The organization of this paper is as follows. In Sec. 2, we explain how to construct the effective spin and pseudospin Hamiltonian of perovskite-type Ti oxides. In Sec. 3 , numerical results calculated by utilizing a mean field approximation are presented. Sec. 4 is devoted to the summary and conclusions. A short version of this paper has been published ${ }^{21)}$, but this paper contains additional and more detailed results. In addition, in the previous paper, we simulated the $\mathrm{GdFeO}_{3}$-type distortion by rotating the $\mathrm{TiO}_{6}$ octahedra around the axes in the $a b$ plane, but in this paper, the distortion is simulated in a more realistic way.

\section{§2. Formalism}

We start with the multiband $d-p$ model in which the full degeneracies of Ti $3 d$ and $\mathrm{O} 2 p$ orbitals as well as the on-site Coulomb and exchange interactions are taken into account. The Hamiltonian is given by

$$
H^{d p}=H_{d 0}+H_{p}+H_{t d p}+H_{t p p}+H_{\mathrm{on}-\mathrm{site}}
$$

with

$$
\begin{aligned}
& H_{d 0}=\sum_{i, \gamma, \sigma} \varepsilon_{d}^{0} d_{i, \gamma, \sigma}^{\dagger} d_{i, \gamma, \sigma}, \\
& H_{p}=\sum_{j, l, \sigma} \varepsilon_{p} p_{j, l, \sigma}^{\dagger} p_{j, l, \sigma}, \\
& H_{t d p}=\sum_{i, \gamma, j, l, \sigma} t_{i \gamma, j l}^{d p} d_{i, \gamma, \sigma}^{\dagger} p_{j, l, \sigma}+\text { h.c., } \\
& H_{t p p}=\sum_{j, l, j^{\prime}, l^{\prime}, \sigma} t_{j l, j^{\prime} l^{\prime}}^{p p} p_{j, l, \sigma}^{\dagger} p_{j^{\prime}, l^{\prime}, \sigma}+\text { h.c. }, \\
& H_{\text {on-site }}=H_{u}+H_{u^{\prime}}+H_{j}+H_{j^{\prime}},
\end{aligned}
$$

where $d_{i, \gamma, \sigma}^{\dagger}$ is a creation operator of an electron with spin $\sigma(=\uparrow, \downarrow)$ in the $3 d$ orbital $\gamma$ at Ti site $i$ and $p_{j, l, \sigma}^{\dagger}$ is a creation operator of an electron with spin $\sigma(=\uparrow, \downarrow)$ in the $2 p$ orbital $l$ at oxygen site $j$. Here, we choose the representation of the fourfold symmetry in the $d$-type JT distortion as the basis of $3 d$ orbitals at each site, namely, $x y, y z, z x, 3 y^{2}-r^{2}$ and $z^{2}-x^{2}$ at site 1 and site 3 and $x y, y z, z x, 3 x^{2}-r^{2}$ and $y^{2}-z^{2}$ at site 2 and site 4 (see Fig. 3). $H_{d 0}$ and $H_{p}$ stand for the bare level energy of Ti $3 d$ and $\mathrm{O} 2 p$ orbitals, respectively. $H_{t d p}$ and $H_{t p p}$ are $d-p$ and $p-p$ hybridization term, respectively. The term $H_{\text {on-site }}$ represents on-site $d$ - $d$ Coulomb interactions. $t_{i \gamma, j l}^{d p}$ and $t_{j l, j^{\prime} l^{\prime}} p p$ are nearest-neighbor $d-p$ transfer and $p-p$ transfer given in terms of Slater-Koster parameters $V_{p d \pi}, V_{p d \sigma}, V_{p p \pi}$ and $V_{p p \sigma}$. $H_{\text {on-site }}$ term consists of the following four contributions:

$$
\begin{aligned}
& H_{u}=\sum_{i, m} u d_{i, m, \uparrow}^{\dagger} d_{i, m, \uparrow} d_{i, m, \downarrow}^{\dagger} d_{i, m, \downarrow}, \\
& H_{u^{\prime}}=\sum_{i, m>m^{\prime}, \sigma, \sigma^{\prime}} u^{\prime} d_{i, m, \sigma}^{\dagger} d_{i, m, \sigma} d_{i, m^{\prime}, \sigma^{\prime}}^{\dagger} d_{i, m^{\prime}, \sigma^{\prime}}
\end{aligned}
$$



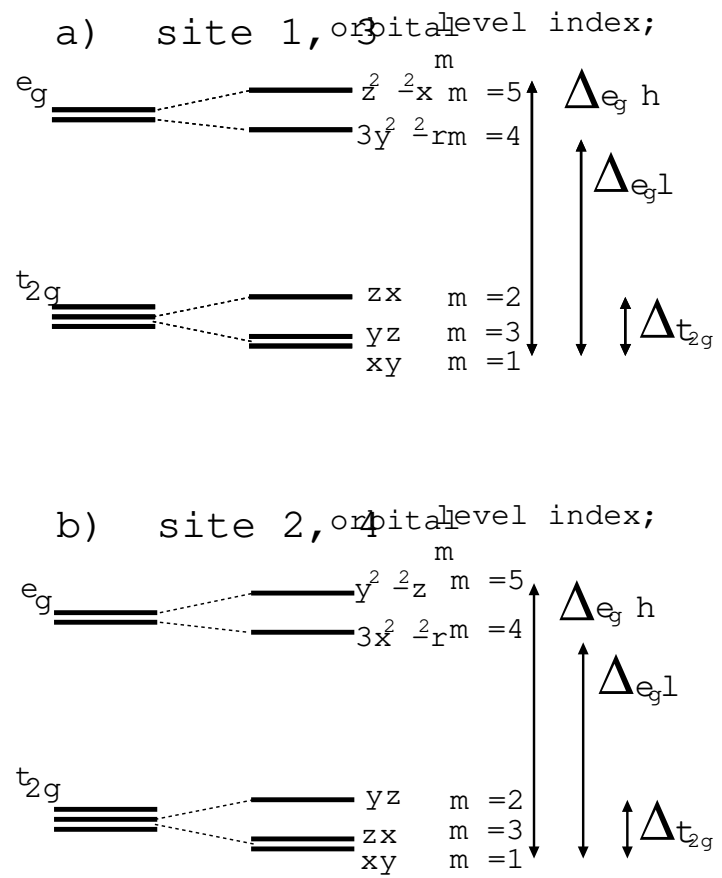

Fig. 3. $3 d$-level splitting in the $d$-type JT distortion: The ways of the level splittings are different between sites 1,3 and sites 2, 4. Fig. a) shows that in sites 1 and 3 and Fig. b) shows that in sites 2 and 4 .

$$
\begin{aligned}
& H_{j}=\sum_{i, m>m^{\prime} \sigma, \sigma^{\prime}} j d_{i, m, \sigma}^{\dagger} d_{i, m^{\prime}, \sigma} d_{i, m^{\prime}, \sigma^{\prime}}^{\dagger} d_{i, m, \sigma^{\prime}}, \\
& H_{j^{\prime}}=\sum_{i, m \neq m^{\prime}} j^{\prime} d_{i, m, \uparrow}^{\dagger} d_{i, m^{\prime}, \uparrow} d_{i, m, \downarrow}^{\dagger} d_{i, m^{\prime}, \downarrow},
\end{aligned}
$$

where $H_{u}$ and $H_{u^{\prime}}$ are the intra- and inter-orbital Coulomb interactions and $H_{j}$ and $H_{j^{\prime}}$ denote the exchange interactions. The term $H_{j}$ is the origin of the Hund's rule coupling which strongly favors the spin alignment in the same direction on the same atoms. The term $H_{j^{\prime}}$ gives the $\uparrow \downarrow$-pair transfer between the $3 d$-orbitals on the same Ti atom. These interactions are expressed by using Kanamori parameters, $u, u^{\prime}, j$ and $j^{\prime}$ which satisfy the following relations ${ }^{22,23)}$;

$$
\begin{aligned}
u & =U+\frac{20}{9} j, \\
u^{\prime} & =u-2 j, \\
j & =j^{\prime} .
\end{aligned}
$$

Here, $U$ gives a magnitude of the multiplet-averaged $d$ $d$ Coulomb interaction. The charge-transfer energy $\Delta$, which describes the energy difference between occupied O $2 p$ and unoccupied Ti $3 d$ levels, is defined by using $U$ and energies of the bare Ti $3 d$ and O $2 p$ orbitals $\varepsilon_{d}^{0}$ and $\varepsilon_{p}$ as follows,

$$
\Delta=\varepsilon_{d}^{0}+U-\varepsilon_{p}
$$

since the characteristic unoccupied $3 d$ level energy on the singly occupied Ti site is $\varepsilon_{d}^{0}+U$. The values of $\Delta$, $U$ and $V_{p d \sigma}$ are estimated by the cluster-model analyses of valence-band and transition-metal $2 p$ core-level photoemission spectra ${ }^{25,26)}$. We take the values of these parameters as $\Delta=7.0 \mathrm{eV}, U=4.0 \mathrm{eV}, V_{p d \sigma}=-2.2$ $\mathrm{eV}$ and $j=0.64 \mathrm{eV}$ throughout the present calculation. The ratio $V_{p d \sigma} / V_{p d \pi}$ is fixed at -2.16 , and $V_{p p \sigma}$ and $V_{p p \pi}$ at $0.60 \mathrm{eV}$ and $-0.15 \mathrm{eV}$, respectively ${ }^{24)}$. The effects of the $\mathrm{GdFeO}_{3}$-type distortion are considered through the $d-p$ transfer integrals which is defined by using the Slater-Koster's parameters ${ }^{27}$ ). The effects of the $d$-type JT distortion are also considered. The magnitude of the distortion can be denoted by the ratio $\left[V_{p d \sigma}^{s} / V_{p d \sigma}^{l}\right]^{1 / 3}$; here, $V_{p d \sigma}^{s}$ and $V_{p d \sigma}^{l}$ are the transfer integrals for the shorter and longer Ti-O bonds. The value for $\mathrm{YTiO}_{3}$ estimated using Harrison's rule ${ }^{24)}$ takes $~ 1.036$. In order to reveal the nature of the magnetic phase transition and the origin of the rapid suppression of $T_{N}$, we focus on the situation near the phase boundary between AFM and FM phases. The value of ratio $\left[V_{p d \sigma}^{s} / V_{p d \sigma}^{l}\right]^{1 / 3}$ is fixed at 1.030, which is expected to be realized near the phase boundary under the assumption of linear decrease as a function of the bond angle from $1.036\left(\mathrm{YTiO}_{3}\right)$ to $1.00\left(\mathrm{LaTiO}_{3}\right)$. Under the JT distortion, the $t_{2 g}$ levelsplitting energy $\Delta_{t_{2 g}}$ is estimated to be $0.050 \mathrm{eV}$. Since the $t_{2 g}$ level-splitting due to the spin-orbit interaction is sufficiently small in comparison with $\Delta_{t_{2 g}}$, we neglect the spin-orbit interaction through the present calculations.

In the path-integral formalism, the expression of the partition function is given by

$Z=\int \mathcal{D} \bar{d}_{i, \gamma, \sigma} \mathcal{D} d_{i, \gamma, \sigma} \mathcal{D} \bar{p}_{j, l, \sigma} \mathcal{D} p_{j, l, \sigma} \exp \left[-\int_{0}^{\beta} \mathrm{d} \tau L(\tau)\right]$,

with

$$
\begin{aligned}
L(\tau)=H^{d p}(\tau) & +\sum_{i, \gamma, \sigma} \bar{d}_{i, \gamma, \sigma}\left(\partial_{\tau}-\mu\right) d_{i, \gamma, \sigma} \\
& +\sum_{j, l, \sigma} \bar{p}_{j, l, \sigma}\left(\partial_{\tau}-\mu\right) p_{j, l, \sigma}
\end{aligned}
$$

where $\tau$ denotes the imaginary time introduced in the path-integral formalism and $\bar{d}_{i, \gamma, \sigma}, d_{i, \gamma, \sigma}, \bar{p}_{j, l, \sigma}$ and $p_{j, l, \sigma}$ are the Grassman-variables corresponding to the operators $d_{i, \gamma, \sigma}^{\dagger}, d_{i, \gamma, \sigma}, p_{j, l, \sigma}^{\dagger}$ and $p_{j, l, \sigma}$, respectively. By using the Matsubara-frequency representation:

$$
\begin{aligned}
d_{i, \gamma, \sigma}(\tau) & =\frac{1}{\sqrt{\beta}} \sum_{\omega_{n}} d_{i, \gamma, \sigma}\left(\omega_{n}\right) e^{-i \omega_{n} \tau} \\
p_{j, l, \sigma}(\tau) & =\frac{1}{\sqrt{\beta}} \sum_{\omega_{n}} p_{j, l, \sigma}\left(\omega_{n}\right) e^{-i \omega_{n} \tau},
\end{aligned}
$$

we have

$$
\begin{aligned}
Z=\int & \mathcal{D} \bar{d}_{i, \gamma, \sigma}\left(\omega_{n}\right) \mathcal{D} d_{i, \gamma, \sigma}\left(\omega_{n}\right) \mathcal{D} \bar{p}_{j, l, \sigma\left(\omega_{n}\right)} \mathcal{D} p_{j, l, \sigma}\left(\omega_{n}\right) \\
& \times \exp \left[-\sum_{\omega_{n}} L\left(\omega_{n}\right)\right]
\end{aligned}
$$

with

$$
L\left(\omega_{n}\right)=\sum_{i, \gamma, \sigma} \bar{d}_{i, \gamma, \sigma}\left(-i \omega_{n}+\varepsilon_{d}^{0}-\mu\right) d_{i, \gamma, \sigma}
$$




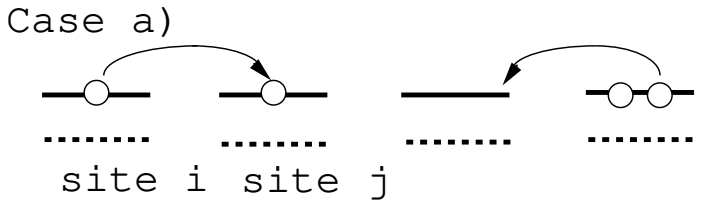

\section{Case b)}

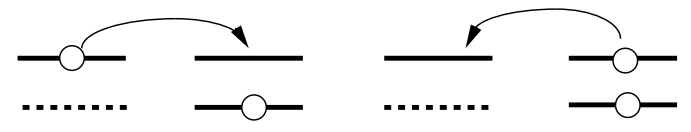

Fig. 4. Characteristic perturbational processes of a $3 d$ electron in two examples. Case a) contains the intermediate states in which an orbital occupied by two electrons with opposite spins in the process. Case b) contains only the states in which an orbital occupied by one electron. The energy takes $\varepsilon_{d}^{0}+u$ in Case a) and $\varepsilon_{d}^{0}+u^{\prime}$ in Case b) at the intermediate states.

$$
\begin{aligned}
& +\sum_{j, l, \sigma} \bar{p}_{j, l, \sigma}\left(-i \omega_{n}+\varepsilon_{p}-\mu\right) p_{j, l, \sigma} \\
& +\sum_{i, \gamma, j, l, \sigma} t_{i m, j l}^{d p} \bar{d}_{i, \gamma, \sigma} p_{j, l, \sigma}+\text { c.c. } \\
& +\sum_{j, l, j^{\prime}, l^{\prime} \sigma} t_{j l, j^{\prime} l^{\prime}}^{p p} \bar{p}_{j, l, \sigma} p_{j^{\prime}, l^{\prime}, \sigma}+\text { c.c. } \\
& +H_{\text {on-site }}\left(\omega_{n}\right) .
\end{aligned}
$$

After integrating over the $\bar{p}$ and $p$, the partition function is rewritten as

$$
Z=\int \mathcal{D} \bar{d}_{i, \gamma, \sigma}\left(\omega_{n}\right) \mathcal{D} d_{i, \gamma, \sigma}\left(\omega_{n}\right) \exp \left[-\sum_{\omega_{n}} L_{d}\left(\omega_{n}\right)\right]
$$

where

$$
\begin{gathered}
L_{d}\left(\omega_{n}\right)=\sum_{i, \gamma, \sigma} \bar{d}_{i, \gamma, \sigma}\left(-i \omega_{n}+\varepsilon_{d}^{0}-\mu\right) d_{i, \gamma, \sigma} \\
+\sum_{\omega_{n}} \sum_{i, \gamma, i^{\prime}, \gamma^{\prime}, \sigma} \sum_{j, l, j^{\prime}, l^{\prime}} \bar{d}_{i, \gamma, \sigma}\left[t_{i \gamma, j l}^{d p}\left(H_{j l, j^{\prime} l^{\prime}}^{-1}\left(i \omega_{n}\right)\right) t_{i^{\prime} \gamma^{\prime}, j^{\prime} l^{\prime}}^{d p}\right] d_{i^{\prime}, \gamma^{\prime}, \sigma} \\
+H_{\text {on-site }}\left(\omega_{n}\right) .
\end{gathered}
$$

Here, a matrix $H_{j l, j^{\prime} l^{\prime}}\left(i \omega_{n}\right)$ takes the form

$$
H_{j l, j^{\prime} l^{\prime}}\left(i \omega_{n}\right)=-\left(-i \omega_{n}+\left(\varepsilon_{p}-\mu\right)\right) \delta_{j l ; j^{\prime} l^{\prime}}-t_{j l, j^{\prime} l^{\prime}}^{p p}
$$

Substituting $i \omega_{n}$ in $H_{j l, j^{\prime} l^{\prime}}^{-1}\left(i \omega_{n}\right)$ with characteristic energies of a $3 d$ electron, we can obtain the expressions of the effective $d-d$ transfers and $3 d$ level energies as follows;

$$
\begin{gathered}
t_{i \gamma, i^{\prime} \gamma^{\prime}}^{d d}=\left\{\begin{array}{c}
\sum_{j, l, j^{\prime}, l^{\prime}} H_{j l, j^{\prime} l^{\prime}}^{-1}\left(\varepsilon_{d}^{0}+u-\mu\right) t_{i \gamma, j l}^{d p} t_{i^{\prime} \gamma^{\prime}, j^{\prime} l^{\prime}}^{d p} \\
\text { for Case a) } \\
\sum_{j, l, j^{\prime}, l^{\prime}} H_{j l, j^{\prime} l^{\prime}}^{-1}\left(\varepsilon_{d}^{0}+u^{\prime}-\mu\right) t_{i \gamma, j l}^{d p} t_{i^{\prime} \gamma^{\prime}, j^{\prime} l^{\prime}}^{d p} \\
\text { for Case b) }
\end{array}\right. \\
\varepsilon_{d i, \gamma}=\varepsilon_{d}^{0}+\sum_{j, l, j^{\prime}, l^{\prime}} H_{j l, j^{\prime} l^{\prime}}^{-1}\left(\varepsilon_{d}^{0}+U-\mu\right) t_{i \gamma, j l}^{d p} t_{i \gamma, j^{\prime} l^{\prime}}^{d p} .
\end{gathered}
$$

The case a) denotes the processes which contain the doubly-occupied-orbital states before or after the electron transfer to a neighboring site. On the other hand, the case b) denotes the processes with no doublyoccupied-orbital states (see Fig. 4). As a result, the "effective" multiband Hubbard Hamiltonian derived from the multiband $d-p$ model has the form;

$$
H^{\mathrm{mH}}=H_{d}^{\mathrm{mH}}+H_{t d d}^{\mathrm{mH}}+H_{\mathrm{on}-\mathrm{site}},
$$

with

$$
\begin{aligned}
& H_{d}^{\mathrm{mH}}=\sum_{i, m, \sigma} \varepsilon_{d i, m} d_{i, m, \sigma}^{\dagger} d_{i, m, \sigma}, \\
& H_{t d d}^{\mathrm{mH}}=\sum_{i, m, i^{\prime}, m^{\prime}, \sigma} t_{i m, i^{\prime} m^{\prime}}^{d d} d_{i, m, \sigma}^{\dagger} d_{i^{\prime}, m^{\prime}, \sigma}+\text { h.c. }, \\
& H_{\text {on-site }}=H_{u}+H_{u^{\prime}}+H_{j}+H_{j^{\prime}},
\end{aligned}
$$

By $H_{d}^{\mathrm{mH}}$, we express the level energies of Ti $3 d$ orbitals under the influence of the crystal fields in the $d$-type JT distortion with

$$
\varepsilon_{d i, m}= \begin{cases}\varepsilon_{d l} & \text { for } m=1,3 \\ \varepsilon_{d l}+\Delta_{t_{2 g}} & \text { for } \quad m=2 \\ \varepsilon_{d l}+\Delta_{e_{g} l} l & \text { for } m=4 \\ \varepsilon_{d l}+\Delta_{e_{g} h} & \text { for } \quad m=5\end{cases}
$$

Here, $m=1,3$ are lower $t_{2 g}$ levels, $m=2$ is a higher $t_{2 g}$ level and $m=4$ and $m=5$ are lower and higher $e_{g}$ levels, respectively. The $\Delta_{t_{2 g}}, \Delta_{e_{g} h}$ and $\Delta_{e_{g} l}$ denote the level-splitting energies measured from lower $t_{2 g}$ level as shown in Fig. 3. It should be noted that the same indices of energy levels at different sites do not necessarily correspond to the orbitals with the same symmetry. $H_{t d d}^{\mathrm{mH}}$ is a $d$ - $d$ super-transfer term.

Among the energy parameters in the multiband Hubbard Hamiltonian, the on-site Coulomb interactions have the largest energy scale. Since the electron filling of the present compounds is kept at $d^{1}$ configuration on average, the on-site Coulomb interactions drive the compounds to the Mott insulating state with suppressed charge fluctuations. Therefore, starting with the multiband Hubbard Hamiltonian, we can derive an effective Hamiltonian in the low-energy region on the subspace of states only with singly occupied $t_{2 g}$ orbitals at each transition-metal site by utilizing a second-order perturbation theory. The states of $3 d$ electron localized at the transition-metal sites can be represented by two quantum numbers, the $z$-component of the spin $S_{z}$ and the number of the occupied orbitals. When one of the twofold lower $t_{2 g}$ orbitals is occupied at each site, we can describe the electronic states using a spin- $1 / 2$ operators, which we call the pseudospin $\tau$. We can describe the occupied energy-level 1 by a quantum number $\tau_{z}=-1 / 2$, and level 3 by $\tau_{z}=+1 / 2$. We follow an approach similar to the well-known Kugel-Khomskii formulation ${ }^{1-4)}$. We express the $3 d$ electron operators in terms of $\mathrm{S}$ and $\boldsymbol{\tau}$ to arrive at the effective spin and pseudospin Hamiltonian:

$$
H_{\mathrm{eff}}=\tilde{H}_{d}^{\mathrm{mH}}+H_{t_{2 g}}+H_{e_{g}}
$$

where

$$
\tilde{H}_{d}^{\mathrm{mH}}=\sum_{i, \sigma, m,=1,2,3} \varepsilon_{d i, m} d_{i, m, \sigma}^{\dagger} d_{i, m, \sigma} .
$$

The first term $\tilde{H}_{d}^{\mathrm{mH}}$ is obtained from the zeroth-order 
perturbational processes. The second term $H_{t_{2 g}}$ is obtained from the second-order perturbational processes whose intermediate states contain only $t_{2 g}$-orbital degrees of freedom. The third term $H_{e_{g}}$ is obtained from the second-order perturbational processes whose intermediate states contain $e_{g}$-orbital degrees of freedom. In this Hamiltonian, the exchange interactions between neighboring spins and orbitals are characterized by the energies in the intermediate states in the perturbational processes. The spin configuration in the system is determined by the competition of the perturbational energy gains which depend on the orbital states and amplitudes of the anisotropic transfer-integrals. Moreover, in this Hamiltonian, the spin and orbital configurations are not determined independently. The terms $H_{t_{2 g}}$ and $H_{e_{g}}$ are described by products of spin and pseudospin operators since the second-order perturbational processes simultaneously change both spin and orbital states in adjacent sites. In this sense, spin and orbital degrees of freedom strongly couple with each other.

In this Hamiltonian, the $e_{g}$-orbital degrees of freedom are taken into account as virtual states of perturbational processes. It is considered that if the energy splitting between $t_{2 g}$ and $e_{g}$ levels $\left(\Delta_{e_{g}}\right)$ is sufficiently large relative to that due to the JT distortion or a characteristic energy scale of low-energy excitations, the relevant electronic orbitals for low-energy excitations or ground-state properties are the $t_{2 g}$ orbitals, and the $e_{g}$-orbital degrees of freedom are negligible. However, in perovskite-type Ti oxides, this energy splitting is rather small relative to the other perovskite-type transition-metal oxides since the charge-transfer energy is relatively large. We can approximately estimate the difference $\Delta_{e_{g}}$ as follows,

$$
\Delta_{e_{g}} \sim 3 \frac{V_{p d \sigma}^{2}}{\Delta}-4 \frac{V_{p d \pi}^{2}}{\Delta},
$$

where $V_{p d \sigma}$ and $V_{p d \pi}$ are the Slater-Koster parameters for the $p$ - $d$ transfer. The expression of Eq. (33) can be easily obtained by Slater and Koster's relations ${ }^{24,27)}$. In $R \mathrm{TiO}_{3}$ case, this value takes about $1.4 \mathrm{eV}$. The order of energy reduction due to the virtual transfer mediated by a singly-occupied $t_{2 g}$ state is given by $\frac{t^{2}}{u}=\frac{t^{2}}{\left(u^{\prime}+2 j\right)}$ and that mediated by an unoccupied $e_{g}$ state is $\frac{t^{2}}{\left(u^{\prime}+\Delta_{e g}\right)}$. Since the values of $\Delta_{e_{g}}$ and $2 j$ are comparable in $R \mathrm{TiO}_{3}$, $\frac{t^{2}}{\left(u^{\prime}+\Delta_{e_{g}}\right)}$-terms are not negligible compared to the $\frac{t^{2}}{u}$ terms. Hence, the $e_{g}$-orbital degrees of freedom cannot be neglected even in the low-energy region so that we take them into consideration as virtual states of the perturbational processes. In general, the charge-transfer energy $\Delta$ gradually increases as the atomic number of the transition-metal decreases.

In addition, we have also examined the effective Hamiltonian on the subspace of states in which an electron occupies not only twofold lower $t_{2 g}$ levels but also a higher $t_{2 g}$ level in the JT distortion at each site by replacing with a pseudospin representation whose magnitude takes 1. However, in the large JT distortion as $\left[V_{p d \sigma}^{s} / V_{p d \sigma}^{l}\right]^{1 / 3}=1.030$ in the present case, the occupancy of the higher $t_{2 g}$ level is close to zero so that the results are not changed from those obtained by using the

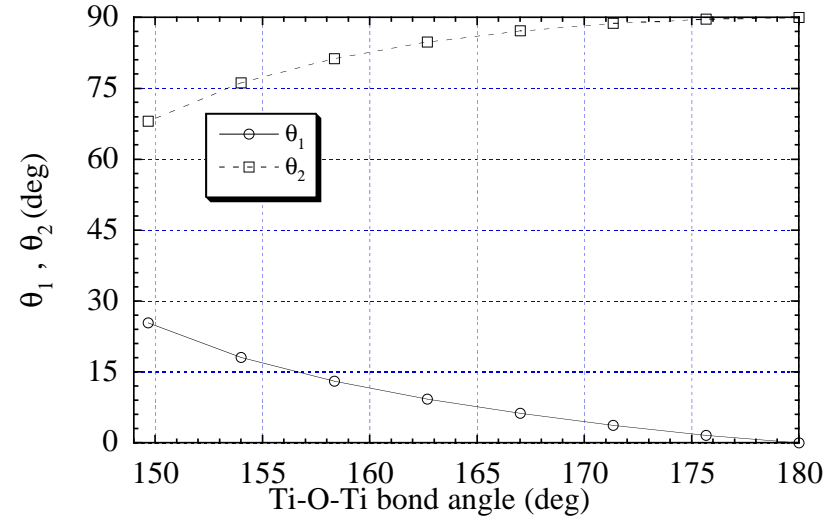

Fig. 5. The orbital structure in the FM1 solution as a function of the Ti-O-Ti bond angle. Without the $\mathrm{GdFeO}_{3}$-type distortion, a complete $(y z, x y, x y, z x)$-type orbital order is realized.

Hamiltonian Eq. (31).

\section{§3. Numerical Results and Discussions}

In this section, we present the numerical results calculated by applying a mean-field approximation to the effective spin and pseudospin Hamiltonian introduced in the previous section. We have introduced the following averages $\left\langle S_{\gamma_{1}}\right\rangle,\left\langle\tau_{\gamma_{1}}\right\rangle$ and $\left\langle S_{\gamma_{1}} \tau_{\gamma_{2}}\right\rangle$ with $\gamma_{1}, \gamma_{2}=x, y$ and $z$ as the mean fields at each site in the $\mathrm{GdFeO}_{3}$-type unit cell, which are to be determined self-consistently.

We have calculated the total energies of various spin and orbital configurations as functions of the bond angle. Without the $\mathrm{GdFeO}_{3}$-type distortion or in the small distortion region $\left(\angle \mathrm{Ti}-\mathrm{O}-\mathrm{Ti}=180^{\circ} \sim 151^{\circ}\right)$, FM solution with $(y z, x y, x y, z x)$-type orbital ordering in which site $1,2,3$ and 4 are dominantly occupied by $y z, x y, x y$, and $z x$, respectively (FM1 solution) is stabilized. We can specify the orbital state realized in the FM1 solution by using two angles $\theta_{1}$ and $\theta_{2}$ as follows,

$$
\begin{array}{ll}
\text { site1; } & \cos \theta_{1}\left|y z>+\sin \theta_{1}\right| x y>, \\
\text { site } 2 ; & \cos \theta_{2}\left|z x>+\sin \theta_{2}\right| x y>, \\
\text { site } 3 ; & -\cos \theta_{2}\left|y z>+\sin \theta_{2}\right| x y>, \\
\text { site } 4 ; & -\cos \theta_{1}\left|z x>+\sin \theta_{1}\right| x y>.
\end{array}
$$

In Fig. 5, the angles $\theta_{1}$ and $\theta_{2}$ are plotted as functions of the Ti-O-Ti bond angle. Without the $\mathrm{GdFeO}_{3}$-type distortion $\left(\angle \mathrm{Ti}-\mathrm{O}-\mathrm{Ti}=180^{\circ}\right)$, complete $(y z, x y, x y, z x)$-type occupation is realized. With increasing the $\mathrm{GdFeO}_{3}$-type distortion, the occupations of the $x y, z x, y z$ and $x y$ orbitals gradually increase at site $1,2,3$ and 4 , respectively. In this orbital ordering, the neighboring occupied orbitals are approximately orthogonal and electron-transfers from the occupied orbitals are restricted to neighboring unoccupied orbitals. This spin and orbital configuration is favored both by transfers and by the exchange interaction $j$ in the small $\mathrm{GdFeO}_{3}$-type distortion. However, it should be noted that in our study, the JT-distortion parameter is fixed at a large value in order to focus on the situation realized near the phase boundary. On the contrary, in $\mathrm{LaTiO}_{3}$ or 


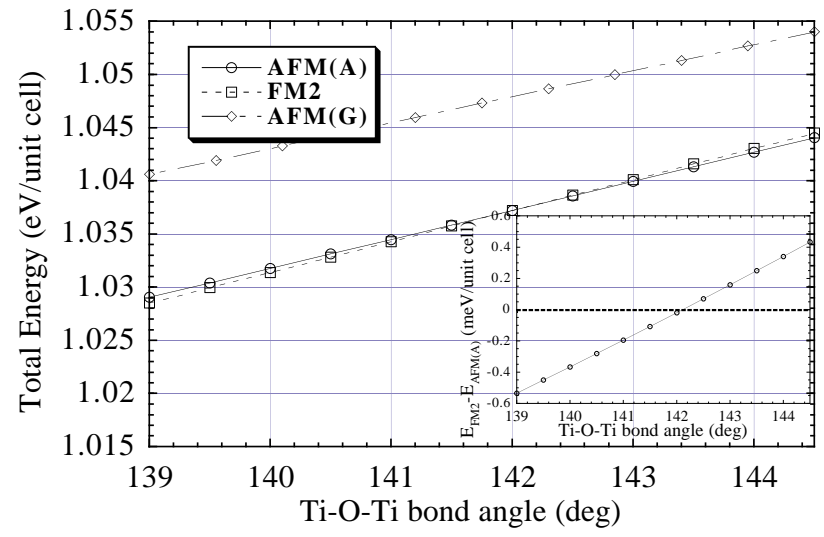

Fig. 6. Total energies of $\operatorname{AFM}(A), \operatorname{FM} 2$ and $\operatorname{AFM}(G)$ solutions near the AFM-FM phase boundary are plotted as functions of the Ti-O-Ti bond angle. Inset shows the energy difference between the $\operatorname{AFM}(\mathrm{A})$ and $\mathrm{FM} 2$ solutions.

in the less distorted region, there is no JT distortion and spin-orbit ground state is considered to be realized. So that, the $\operatorname{AFM}(G)$ state is not reproduced in this calculation even in the less distorted region. In addition, we should note that the FM1 solution which is stabilized in small $\mathrm{GdFeO}_{3}$-type and large JT distortions is not realized in the actual systems. By moderately decreasing the Ti-O-Ti bond angle (increasing the $\mathrm{GdFeO}_{3}$-type distortion), the A-type $\operatorname{AFM}(\operatorname{AFM}(\mathrm{A}))$ solution with another type of orbital ordering is stabilized rather than the FM1 solution around $\angle \mathrm{Ti}-\mathrm{O}-\mathrm{Ti} \sim 151^{\circ}$. With further decreasing of the Ti-O-Ti bond angle, a FM state accompanied by an orbital ordering is stabilized again. The type of the orbital ordering in this FM phase is similar to that in the $\operatorname{AFM}(\mathrm{A})$ phase. Hereafter, we refer to this FM state as FM2. The AFM to FM phase transition observed in the actual system corresponds to this $\operatorname{AFM}(\mathrm{A})$ to FM2 phase transition. In Fig. 6, total energies of the $\operatorname{AFM}(\mathrm{A}), \mathrm{FM} 2$ and $\operatorname{AFM}(\mathrm{G})$ states are plotted near the phase boundary. The $\operatorname{AFM}(\mathrm{G})$ solution has much higher energy relative to other two solutions. The $\operatorname{AFM}(\mathrm{A})$ to FM2 phase transition occurs at $\angle \mathrm{Ti}-\mathrm{O}-\mathrm{Ti} \sim 142^{\circ}$.

We can specify the orbital states realized in the $\operatorname{AFM}(\mathrm{A})$ and FM2 solutions by using the angle $\theta_{\mathrm{AFM}(\mathrm{A})}$ and $\theta_{\mathrm{FM} 2}$ as,

$$
\begin{array}{ll}
\text { site1; } & \cos \theta_{x}\left|x y>+\sin \theta_{x}\right| y z>, \\
\text { site2; } & \cos \theta_{x}\left|x y>+\sin \theta_{x}\right| z x>, \\
\text { site3; } & -\cos \theta_{x}\left|x y>+\sin \theta_{x}\right| y z>, \\
\text { site4; } & -\cos \theta_{x}\left|x y>+\sin \theta_{x}\right| z x>,
\end{array}
$$

where $x=\operatorname{AFM}(\mathrm{A}), \mathrm{FM} 2$. In Fig. 7 , the angles for the $\operatorname{AFM}(\mathrm{A})$ and $\mathrm{FM} 2$ solutions $\left(\theta_{\mathrm{AFM}(\mathrm{A})}, \theta_{\mathrm{FM} 2}\right)$ are plotted. The difference between the $\theta_{\mathrm{AFM}(\mathrm{A})}$ and $\theta_{\mathrm{FM} 2}$ is very small and both take almost the same value $\left(\sim 45^{\circ}\right)$. This indicates that the way of the orbital ordering changes only little through the magnetic phase transition. Hereafter, we refer to the orbital state realized in the $\operatorname{AFM}(\mathrm{A})$ and FM2 phases as $(y z, z x, y z, z x)$-type orbital order.

In Fig. 8, the absolute values of the spin-independent

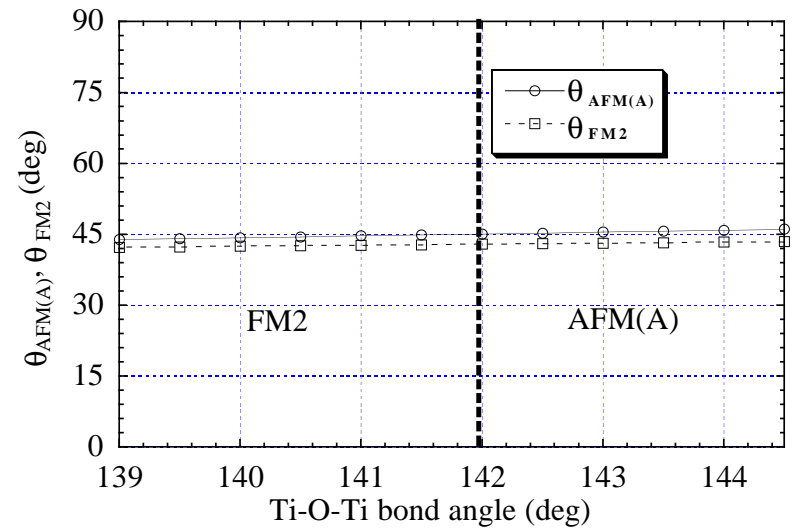

Fig. 7. The orbital structures in the $\operatorname{AFM}(\mathrm{A})$ and FM2 solutions as functions of the Ti-O-Ti bond angle. The difference between those of the two solutions are considerably small.

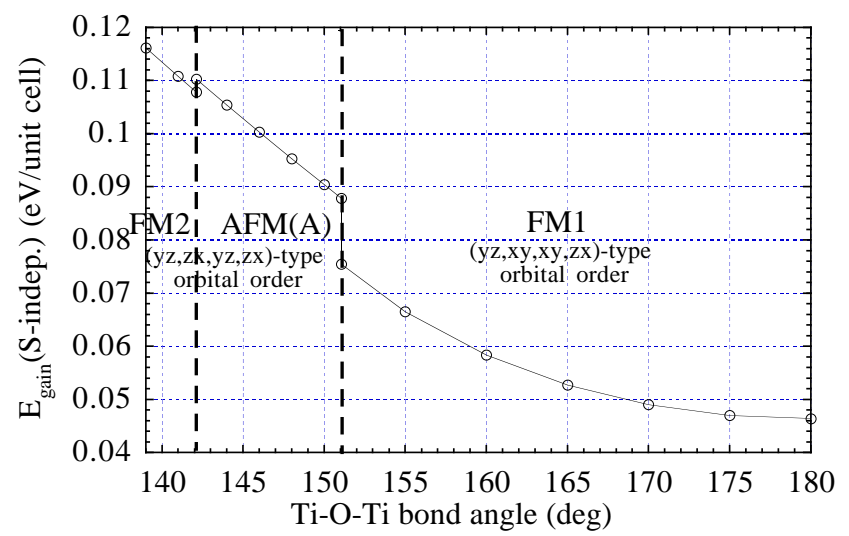

Fig. 8. The absolute values of spin-independent energy gains for stabilized solutions as functions of the $\mathrm{Ti}-\mathrm{O}-\mathrm{Ti}$ bond angle, which are calculated by substituting $S$ with 0 .

energy gains due to the second-order perturbations $\left(E_{\text {gain }}(S\right.$-indep. $\left.)\right)$ are plotted for the stabilized spin and orbital structures. The figure shows that $(y z, x y, x y, z x)$ type to ( $y z, z x, y z, z x)$-type orbital state phase transition accompanied by a large jump of the energy-gain occurs at $151^{\circ}$ and the value increases rapidly as the bond angle decreases in the $(y z, z x, y z, z x)$-type orbital order region. This indicates that the $(y z, z x, y z, z x)$-type orbital order is strongly favored as Ti-O-Ti bond angle is decreased. As the $\mathrm{GdFeO}_{3}$-type distortion increases, the indirect hybridization between the neighboring $t_{2 g}$ orbitals and $e_{g}$ orbitals increases. Since the hybridizations between neighboring $e_{g}$ and $\mathrm{O} 2 p$ orbitals have a $\sigma$-bonding character, amplitudes of the transfers between these orbitals are critically increased by the distortion. Hence, the $e_{g^{-}}$ orbital degrees of freedom becomes indispensable for the stability of the ( $y z, z x, y z, z x)$-type orbital ordering and the orbital ordering is strongly stabilized with increasing the $\mathrm{GdFeO}_{3}$-type distortion because of the large amplitudes of transfers toward neighboring $e_{g}$ orbitals. Actually, within the model which does not contain the $e_{g^{-}}$ orbital degrees of freedom, only the FM solution with 


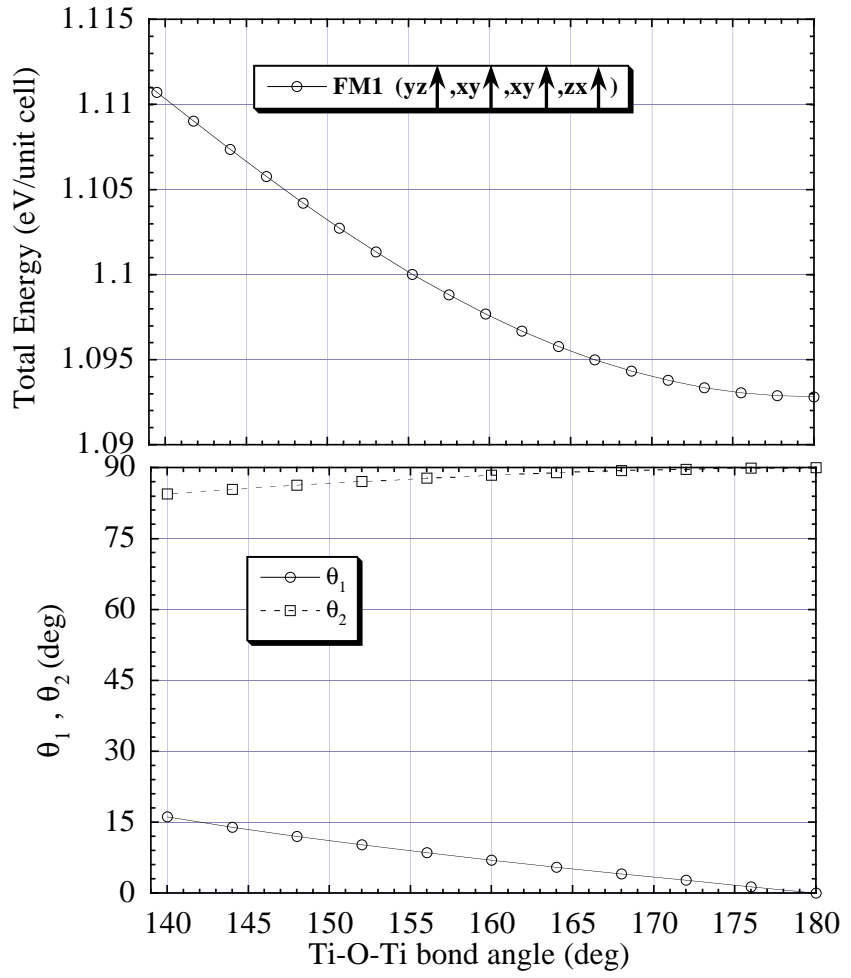

Fig. 9. (Upper panel); The total energy for the FM1 solution calculated by utilizing a Hamiltonian which includes only $t_{2 g}$ orbital degrees of freedom $\left(H_{\text {eff }}=\tilde{H}_{d}^{\mathrm{mH}}+H_{t_{2 g}}\right)$. Within this model, the other spin and orbital configurations do not have any stable solutions. (Lower panel); The orbital structures in this FM1 solution.

( $y z, x y, x y, z x)$-type orbital order (FM1) is stabilized and the $(y z, z x, y z, z x)$-type orbital state does not have any stable solutions. Moreover, the energy of the FM1 state increases as the magnitude of the $\mathrm{GdFeO}_{3}$-type distortion is increased as shown in Fig. 9 (upper panel). This tendency is consistent with the fact that $t_{2 g}$ bandwidth is reduced as the Ti-O-Ti bond angle decreases. The orbital state realized in this FM1 solution can also be specified by utilizing two angles $\theta_{1}$ and $\theta_{2}$ as Eq. (34). The angles $\theta_{1}$ and $\theta_{2}$ are plotted in Fig. 9 (lower panel).

The AFM(A)-to-FM2 phase transition is identified as the transition where the sign of the spin-exchange interaction along the $c$-axis changes from negative to positive while that in the $a b$-plane is constantly negative. The constant FM coupling in the $a b$-plane under the $(y z, z x, y z, z x)$-type orbital state can be easily understood. In the $a b$-plane, the neighboring orbitals are approximately orthogonal to each other. Hence, the FM spin configuration is favored through Hund's rule coupling interaction. However, the emergence of the FM2 phase is not understood straightforwardly since the neighboring orbitals along the $c$-axis are not orthogonal. By considering the transfers from an occupied orbital to neighboring $e_{g}$ orbitals, this is understood schematically as follows. At this stage, based on the fact that the orbital state hardly changes between two phases, we fix the angle $\theta_{\mathrm{AFM}(\mathrm{A})}$ and $\theta_{\mathrm{FM} 2}$ at $45^{\circ}$. Namely, we as- sume that an electron occupies $\frac{1}{\sqrt{2}}(x y+y z), \frac{1}{\sqrt{2}}(x y+z x)$, $\frac{1}{\sqrt{2}}(-x y+y z)$ and $\frac{1}{\sqrt{2}}(-x y+z x)$ at sites $1,2,3$, and 4 , respectively through the phase transition. In addition, we use the representation of the cubic symmetry $x^{2}-y^{2}$ and $3 z^{2}-r^{2}$ for the $e_{g}$ orbitals for intuitive understanding. Let us consider the energy gain of an electron in the $\frac{1}{\sqrt{2}}(x y+y z)$ orbital at site 1 , which is caused by the second-order perturbational processes with respect to the transfers along the $c$-axis (i.e., the transfers between site 1 and site 3$)$. In the large $\mathrm{GdFeO}_{3}$-type distortion, the $\frac{1}{\sqrt{2}}(x y+y z)$ orbital at site 1 mainly hybridizes with the $y z$ and $3 z^{2}-r^{2}$ orbitals at site 3 along the $z$ direction relative to the other orbitals. For example, the transfer amplitudes between $y z$ at site 1 and $z x$ or $x y$ orbitals at site 3 are smaller by as much as $10^{-2}$ than those between $y z$ at site 1 and $y z$ or $3 z^{2}-r^{2}$ orbitals at site 3 . When the $\frac{1}{\sqrt{2}}(-x y+y z)$ orbital at site 3 is occupied by an electron, the second-order perturbational energy gain of an electron in the $\frac{1}{\sqrt{2}}(x y+y z)$ orbital at site 1 depends on the spin configuration between site 1 and site 3 . When the spins of electrons on site 1 and site 3 are antiparallel, the absolute value of the energy gain can be written approximately as follows (see Fig. 10(a)),

$$
\frac{t_{1}^{2}}{u^{\prime}}+\frac{t_{2}^{2}}{u}+\frac{t_{3}^{2}}{u^{\prime}+\Delta_{e_{g}}}
$$

Here, $t_{1}$ represents the transfer between $\frac{1}{\sqrt{2}}(x y+y z)$ at site 1 and $\frac{1}{\sqrt{2}}(x y+y z)$ at site 3 , and $t_{2}$ represents that between $\frac{1}{\sqrt{2}}(x y+y z)$ at site 1 and $\frac{1}{\sqrt{2}}(-x y+y z)$ at site 3 , and $t_{3}$ represents that between $\frac{1}{\sqrt{2}}(x y+y z)$ at site 1 and $3 z^{2}-r^{2}$ at site 3 , and $\Delta_{e_{g}}$ denotes the level-energy difference between $t_{2 g}$ and $e_{g}$ level in the cubic crystal field. On the other hand, when the spins are parallel, transfer to the $\frac{1}{\sqrt{2}}(-x y+y z)$ orbital is forbidden by Pauli's principle but the energies of the intermediate states in which two electrons occupy different orbitals are reduced by the intrasite exchange interaction $j$ (see Fig. 10(b)). Consequently, the absolute value of the energy gain can be written as

$$
\begin{aligned}
\frac{t_{1}^{2}}{u^{\prime}-j} & +\frac{t_{3}^{2}}{u^{\prime}+\Delta_{e_{g}}-j} \\
& \sim \frac{t_{1}^{2}}{u^{\prime}}+\frac{t_{1}^{2} j}{u^{\prime 2}}+\frac{t_{3}^{2}}{u^{\prime}+\Delta_{e_{g}}}+\frac{t_{3}^{2} j}{\left(u^{\prime}+\Delta_{e_{g}}\right)^{2}} .
\end{aligned}
$$

Therefore, the spin configuration between site 1 and site 3 is determined by the competition between the following two energies, $\frac{t_{2}^{2}}{u}$ and $\frac{t_{1}^{2} j}{u^{\prime 2}}+\frac{t_{3}^{2} j}{\left(u^{\prime}+\Delta_{e_{g}}\right)^{2}}$. In Fig. 11 (upper panel), the values of these energies are plotted as functions of the bond angle. As the $\mathrm{GdFeO}_{3}$-type distortion increases, the indirect hybridizations between neighboring $t_{2 g}$ orbitals are decreased and those between neighboring $t_{2 g}$ orbitals and $e_{g}$ orbitals are increased. Consequently, the value of $t_{2}^{2} / u$ is decreased and that of $t_{3}^{2} j /\left(u^{\prime}+\Delta_{e_{g}}\right)^{2}$ is increased, resulting in the crossing of the two energies as the bond angle is decreased. Moreover, since the hybridization between neighboring $e_{g}$ and $t_{2 g}$ orbitals mediated by $\mathrm{O} 2 p$ orbitals (i.e., $t_{3}$ ) becomes to have a $\sigma$-bonding character with the $\mathrm{GdFeO}_{3}$-type distortion, the amplitudes of the $t_{3}$ and $t_{3}^{2} j /\left(u^{\prime}+\Delta_{e_{g}}\right)^{2}$ are 


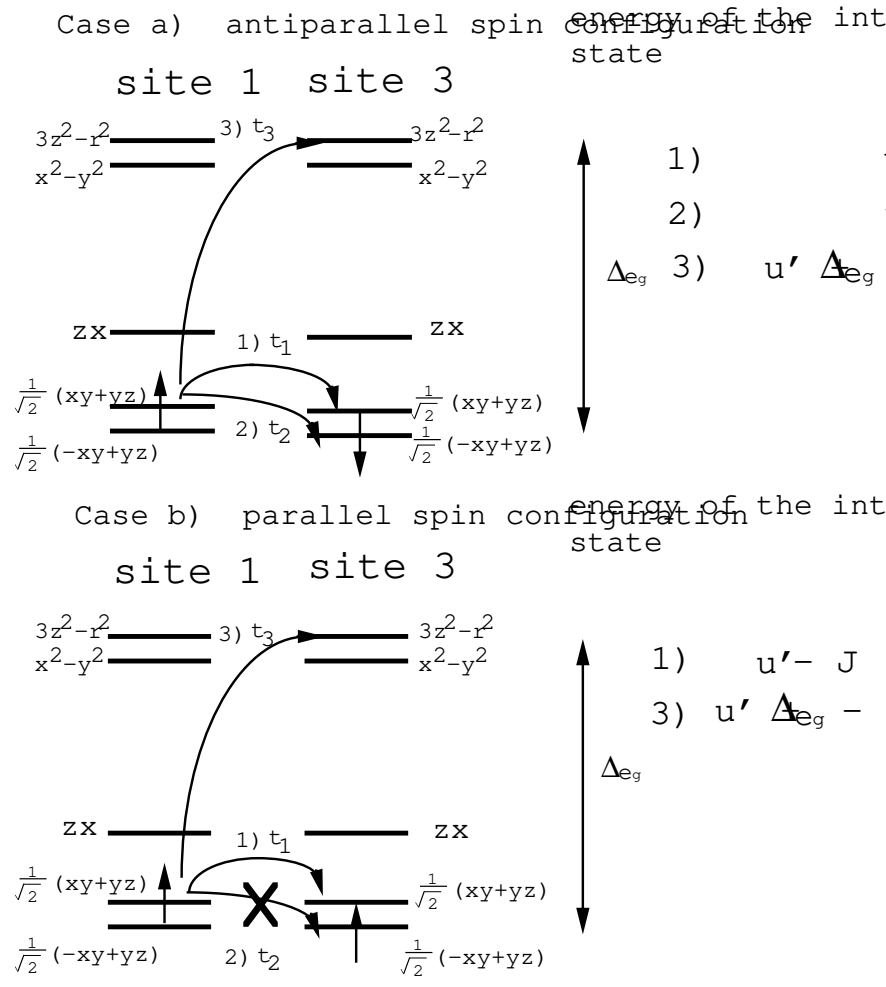

Fig. 10. The second-order perturbational energy gain strongly depends on the spin configuration. Substantial transfers along the $c$-axis for the energy gain are illustrated for both the parallel and antiparallel spin configurations. The angles $\theta_{\mathrm{AFM}(\mathrm{A})}$ and $\theta_{\mathrm{FM} 2}$ are fixed at $45^{\circ}$. The cross symbol $\times$ represents the forbidden transfer.

critically increased.

On the basis of the above discussions, we can well describe this system by the following Heisenberg model as long as the $(y z, z x, y z, z x)$-type orbital order is strongly stabilized and hardly affected by the change in the spin configuration,

$$
H_{\mathrm{Heis}}=J_{\mathrm{Heis}}^{c} \sum_{\langle i, j\rangle}^{c} \boldsymbol{S}_{i} \cdot \boldsymbol{S}_{j}+J_{\text {Heis }}^{a, b} \sum_{\langle i, j\rangle}^{a, b} \boldsymbol{S}_{i} \cdot \boldsymbol{S}_{j}
$$

with

$$
\begin{aligned}
& J_{\text {Heis }}^{a, b}<0, \\
& J_{\text {Heis }}^{c}=4\left(\frac{t_{2}^{2}}{u}-\left(\frac{t_{1}^{2} j}{u^{\prime 2}}+\frac{t_{3}^{2} j}{\left(u^{\prime}+\Delta_{e_{g}}\right)^{2}}\right)\right) .
\end{aligned}
$$

Here, $\sum_{\langle i, j\rangle}^{c}$ denotes the summation over the neighboring spin couplings along the $c$-axis and $\sum_{\langle i, j\rangle}^{a, b}$ in the $a b$-plane. The $\operatorname{AFM}(\mathrm{A})$-to-FM2 phase transition occurs by the change in the sign of $J_{\text {Heis }}^{c}$. Moreover, within this model, the value of $J_{\text {Heis }}^{c}$ decreases from a positive to a negative value continuously as the bond angle is decreased and becomes zero at the phase boundary as shown in Fig. 11 (lower panel). Two-dimensional spin coupling is realized at the phase boundary. Conse-
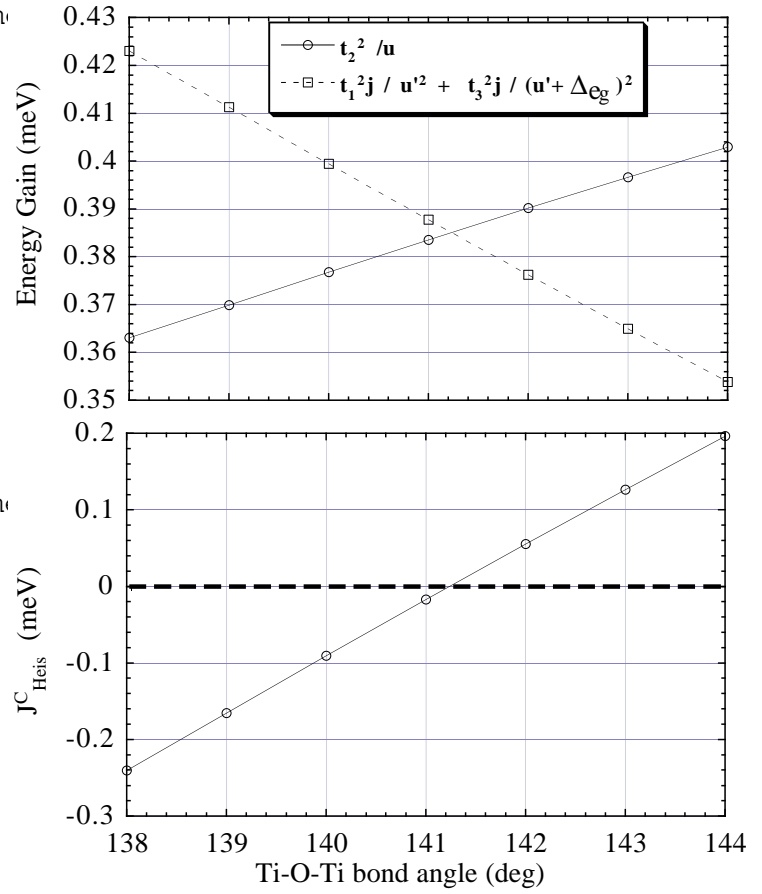

Fig. 11. A characteristic second-order perturbational energy gain due to the transfers along the $c$-axis for the antiparallel spin configuration $t_{2}^{2} / u$ and that for the parallel spin configuration $t_{1}^{2} j / u^{\prime 2}+t_{3}^{2} j /\left(u^{\prime}+\Delta_{e_{g}}\right)^{2}$ are plotted as functions of the Ti-O$\mathrm{Ti}$ bond angle. Two energies are crossing at about $141^{\circ}$. With decreasing the Ti-O-Ti bond angle, the sign of the spin-exchange interaction along the $c$-axis changes from positive to negative continuously at the phase boundary.

quently, the critical temperature at the phase boundary is suppressed to zero, in accordance with Mermin and Wagner's theorem ${ }^{28)}$.

However, the orbital states are actually slightly different between $\operatorname{AFM}(\mathrm{A})$ and $\mathrm{FM} 2$ solutions. In order to investigate how this slight difference in orbital structures affects the spin-exchange interactions along the $c$ axis and those in the $a b$-plane, we estimate the values of $J_{\text {Heis }}^{c}$ and $J_{\text {Heis }}^{a, b}$ as functions of the bond angle for the optimized orbital states of the stabilized spin configuration. $J_{\text {Heis }}^{c}$ and $J_{\text {Heis }}^{a, b}$ are represented as,

$$
\begin{gathered}
J_{\text {Heis }}^{c}=\left(E_{\mathrm{FM}}-E_{\mathrm{AFM}(\mathrm{A})}\right) / 2 S^{2}, \\
2 J_{\text {Heis }}^{a, b}=\left(E_{\mathrm{FM}}-E_{\mathrm{AFM}(\mathrm{C})}\right) / 2 S^{2} .
\end{gathered}
$$

Here, $E_{\mathrm{FM}}, E_{\mathrm{AFM}(\mathrm{A})}$ and $E_{\mathrm{AFM}(\mathrm{C})}$ are the energy gains per unit formula for $\mathrm{FM}, \operatorname{AFM}(\mathrm{A})$ and $\operatorname{AFM}(\mathrm{C})$ spin configurations, respectively, which are due to the secondorder perturbational processes under the orbital states of stabilized solutions. In Fig. 12, the variations of $J_{\text {Heis }}^{c}$ and $J_{\text {Heis }}^{a, b}$ are plotted as functions of the bond angle. While $J_{\text {Heis }}^{a, b}$ constantly has a large negative value $(\sim-2.5 \mathrm{meV}$; ferromagnetic coupling), a positive value of $J_{\text {Heis }}^{c}$ in the relatively large bond-angle region decreases as the bond angle decreases, and suppressed to very small positive value as the $\mathrm{Ti}-\mathrm{O}-\mathrm{Ti}$ bond angle goes to a transition point $\left(\sim 142 \cdot 1^{\circ}\right)$. Then, $J_{\text {Heis }}^{c}$ slightly jumps to a negative value, caused by a small change in the orbital state at the phase boundary. Although a small jump of the 


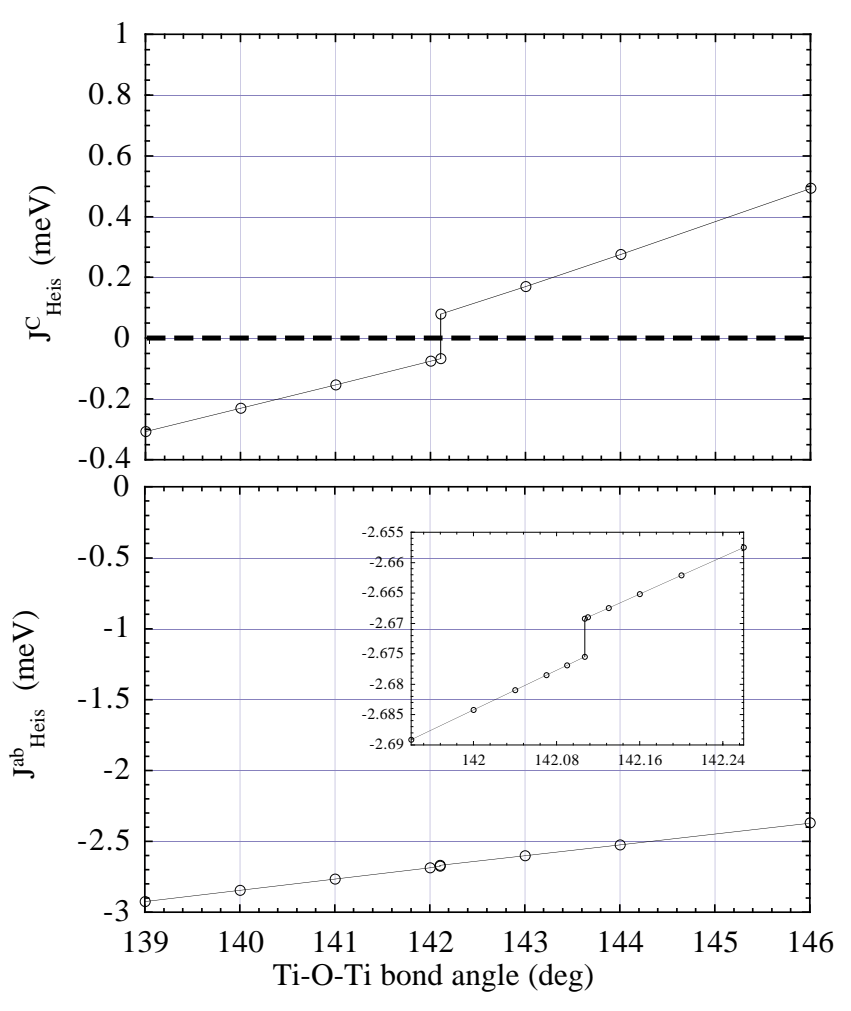

Fig. 12. The value of the spin-exchange interaction along the $c$ axis $\left(J_{\text {Heis }}^{c}\right)$ and that in the $a b$-plane $\left(J_{\text {Heis }}^{a, b}\right)$ are plotted as functions of the Ti-O-Ti bond angle. While a small jump of the $J_{\text {Heis }}^{c}$ value arises by a slight change of the orbital state, the value is suppressed to almost zero at the phase boundary. The inset of the lower panel magnifies the small jump of the $J_{\text {Heis }}^{a, b}$ at the phase boundary.

$J_{\text {Heis }}^{c}\left(\Delta J_{\text {Heis }}^{c}\right)$ from a positive value $(\sim 0.1 \mathrm{meV})$ to a negative one $(\sim-0.1 \mathrm{meV})$ occurs, the absolute values of $J_{\text {Heis }}^{c}$ and $\Delta J_{\text {Heis }}^{c}$ at the phase boundary is sufficiently small relative to that of $J_{\text {Heis }}^{a, b}(\sim 2.7 \mathrm{meV})$. Therefore, we can conclude that the strong two-dimensionality in spin couplings is realized near the phase boundary. At this stage, the scenario of the rapid decrease of $T_{N}$ is as follows: The $\mathrm{GdFeO}_{3}$-type distortion increases the indirect hybridizations between neighboring $t_{2 g}$ and $e_{g}$ orbitals. As a result, ( $y z, z x, y z, z x)$-type orbital order is strongly stabilized by the energy gain due to the transfers almost independently of the spin configuration. Since the orbital structure changes only little through the magnetic phase transition, the spin-exchange interaction along the $c$-axis decreases from a positive value to a negative one almost continuously with increasing the $\mathrm{GdFeO}_{3}$-type distortion, and consequently becomes almost zero $(\sim 0.1 \mathrm{meV})$ at the phase boundary while that in the $a b$-plane remains ferromagnetic at $-2.67 \mathrm{meV}$. This strong two-dimensionality at the phase boundary suppresses the transition temperatures $T_{N}$ and $T_{C}$.

In Fig. 13, we show the magnetic phase diagram as a function of the bond angle under the application of the magnetic field in the spin-ordering direction (z-direction). With increasing the magnetic field, the ground-state $\operatorname{AFM}(\mathrm{A})$ spin configuration changes into FM one at a threshold value of the magnetic field. The

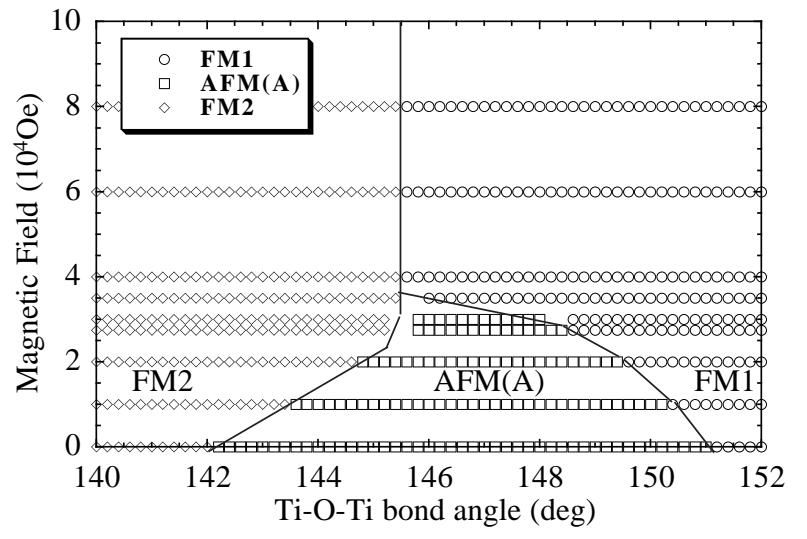

Fig. 13. The magnetic phase diagram under the application of the magnetic field. The value of the JT-distortion-parameter takes 1.030 .

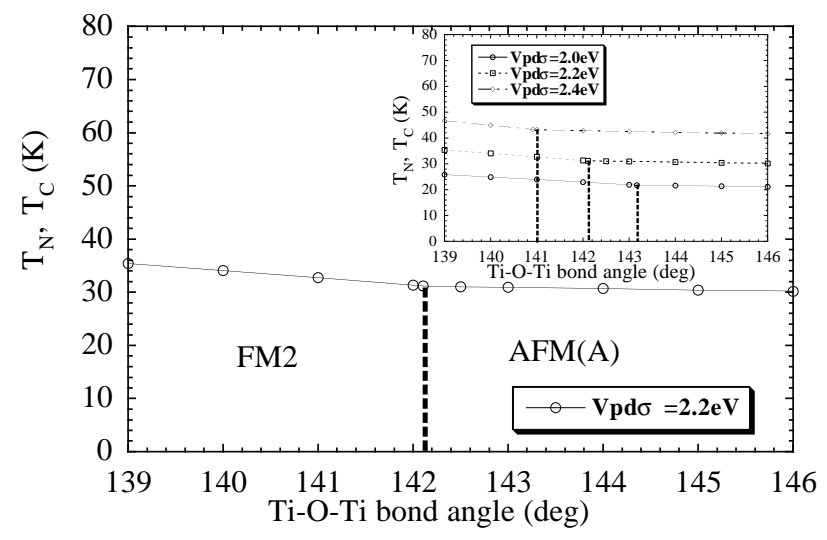

Fig. 14. Magnetic transition temperatures $T_{N}$ and $T_{C}$ calculated by the mean-field approximation in the case $V_{p d \sigma}=2.0 \mathrm{eV}$ are plotted as functions of the Ti-O-Ti bond angle. The inset shows $T_{N}$ and $T_{C}$ in the case of $V_{p d \sigma}=2.0 \mathrm{eV}, 2.2 \mathrm{eV}$ and $2.4 \mathrm{eV}$.

threshold value of the $\operatorname{AFM}(\mathrm{A})$-to-FM2 spin-flip transition driven by the application of the magnetic field linearly decreases as the Ti-O-Ti bond angle decreases. This may reflect the linear behavior of $J_{\text {Heis }}^{c}$ as a function of the bond angle. Although we may expect more complicated behavior in the presence of the magnetic anisotropy in the compounds, the qualitative structure of the phase diagram in Fig. 13 remain valid for small anisotropy.

In Fig. 14, we show the values of $T_{N}$ and $T_{C}$ which are calculated by using the mean-field approximation. A value of $T_{C}$ at $\angle \mathrm{Ti}-\mathrm{O}-\mathrm{Ti}=140^{\circ}$ is in good agreement with the experimental result for $\mathrm{YTiO}_{3}$. However, the suppression of the critical temperature at the FM-AFM phase boundary which is expected to be realized from the two-dimensional character cannot be reproduced within this mean-field calculation. Instead, we can discuss the behavior of the critical temperature qualitatively in the following manner: Near the phase boundary, the spin coupling in this system can be well described by the Heisenberg model given in Eq. (38) since the orbital state is hardly affected by the spin state. Besides, the strong spin-exchange interaction in the $a b$-plane $J_{\text {Heis }}^{a, b}$ 
and the weak interaction along the $c$-axis $J_{\text {Heis }}^{c}$ realize the strong two-dimensionality in the spin coupling;

$$
\left|J_{\text {Heis }}^{c}\right| \ll\left|J_{\text {Heis }}^{a, b}\right| .
$$

At this stage, it is justified to deal with the spin-exchange term along the $c$-axis $H_{\text {Heis }}^{c}$ within the mean-field approximation. Then, we can write the magnetic susceptibility of this system $\chi_{3 D}$ as,

$$
\chi_{3 D}=\frac{\chi_{2 D}}{1-J_{\text {Heis }}^{c} \chi_{2 D}} .
$$

Here, $\chi_{2 D}$ denotes the magnetic susceptibility of the twodimensional spin system described by the Hamiltonian,

$$
H_{\text {Heis }}=J_{\text {Heis }}^{a, b} \sum_{\langle i, j\rangle}^{a, b} \boldsymbol{S}_{i} \cdot \boldsymbol{S}_{j},
$$

Because of the strong spin-exchange interaction in the $a b$-plane, when the system undergoes the magnetic phase transition, the spin correlation in the $a b$-plane is expected to be sufficiently large and the system is to be in the renormalized-classical regime where $\chi_{2 D}$ has a form,

$$
\chi_{2 D} \propto \exp \left(\frac{J_{\text {Heis }}^{a, b}}{T}\right) .
$$

In addition, $J_{\text {Heis }}^{c}$ shows a linear behavior as a function of the angle $\theta-\theta_{\mathrm{c}}$ near the phase boundary;

$$
J_{\text {Heis }}^{c} \propto\left|\theta-\theta_{\mathrm{c}}\right| \text {. }
$$

So that, the critical temperature near the phase boundary which is defined by the divergence of $\chi_{3 D}$ can be written as a function of $\theta-\theta_{\mathrm{c}}$ as follows,

$$
T_{\text {crit. }} \propto \frac{1}{-\ln \left(\left|\theta-\theta_{\mathrm{c}}\right|\right)+\text { const. }} .
$$

This expression implies the rapid suppression of the critical temperature as the system goes to the AFM-FM phase boundary.

Moreover, the large $T_{N}$ values $(\sim 120 \mathrm{~K})$ realized in the small $\mathrm{GdFeO}_{3}$-type distortion or the La-rich region cannot be reproduced within this calculation. We also calculated the magnetic transition temperatures in the case $V_{p d \sigma}=2.0$ and $2.4 \mathrm{eV}$ (see Inset of Fig. 14). Although the values of $T_{C}$ and $T_{N}$ increases as the value of $V_{p d \sigma}$ is increased, the large $T_{N}$ value observed in experiments cannot be reproduced. This indicates that the AFM state realized in the $\mathrm{LaTiO}_{3}$ or in the less distorted region and that near the FM-AFM phase boundary or in the more distorted region are qualitatively different. In the $\operatorname{AFM}(\mathrm{A})$ or $\mathrm{FM} 2$ region, the $(y z, z x, y z, z x)$-type orbital order is stabilized irrespective of the spin structure. Consequently, the energy gain which depends on the spin structure may be rather small, resulting in the effectively small spin-exchange interaction and small $T_{N}$ and $T_{C}$ values. On the contrary, the spin and orbital degrees of freedom in $\mathrm{LaTiO}_{3}$ strongly couple to each other, which may result in the effectively strong spinexchange interaction and large $T_{N}$ value. It may be necessary to take the spin-orbit interaction into consideration to reproduce the behavior of $T_{N}$ in the less distorted region. According to the experimentally observed mag-

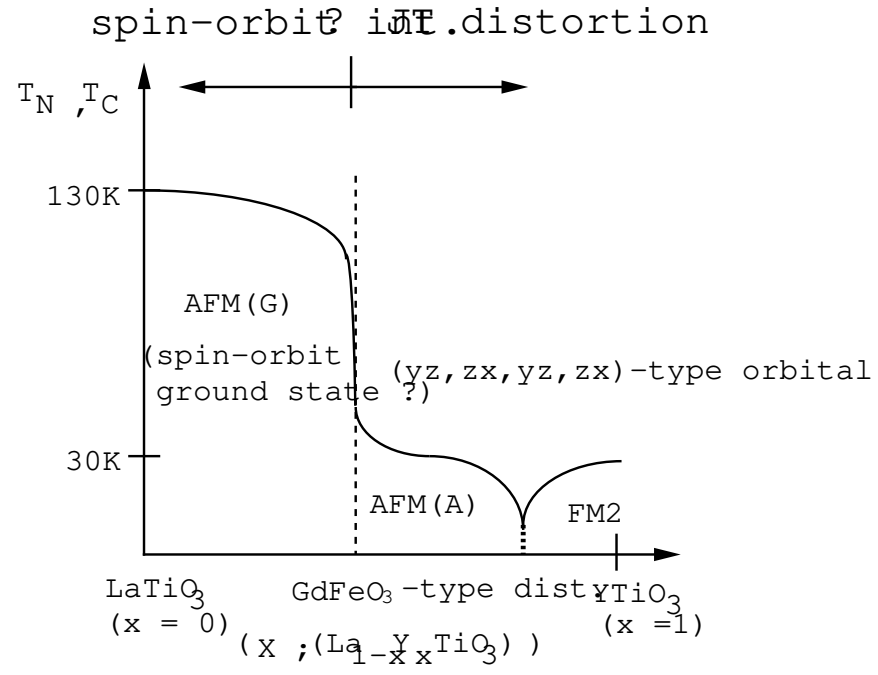

Fig. 15. Schematic magnetic phase diagram. $T_{N}$ takes relatively large value in the $\operatorname{AFM}(G)$ phase realized in the region of the small $\mathrm{GdFeO}_{3}$-type and $d$-type distortions. With increasing the $\mathrm{GdFeO}_{3}$-type distortion, the $\operatorname{AFM}(\mathrm{A})$ phase accompanied by the $(y z, z x, z x, y z)$-type orbital ordering is realized with small $\operatorname{AFM}(\mathrm{A})$ spin coupling along the $c$-axis. On the contrary, the rather strong FM coupling is realized in the $a b$-plane and this drives the strong two dimensional character in this system. This causes the rapid suppression of the $T_{N}$. The AFM spin-coupling along the $c$-axis is decreased almost linearly and strongly suppressed as system goes to the $\operatorname{AFM}(\mathrm{A})$-to-FM2 phase boundary. This causes the second-order like behavior of this AFM to FM phase transition. The JT distortion is relevant to the orbital state in $\operatorname{AFM}(\mathrm{A})$ and $\mathrm{FM} 2$ phases while in the $\operatorname{AFM}(\mathrm{G})$ phase, the spin-orbit interaction is considered to be substantial.

netic phase diagram, $T_{N}$ rapidly decreases from rather large value $(\sim 100 \mathrm{~K})$ around the distortion $\sim 151^{\circ}$. This suggests that the AFM spin coupling along the $c$-axis is already suppressed strongly when the $\operatorname{AFM}(\mathrm{A})$ phase accompanied by the $(y z, z x, y z, z x)$-type orbital state is stabilized with increasing the magnitude of the $\mathrm{GdFeO}_{3}-$ type distortion. Consequently, the schematic magnetic phase diagram can be obtained as shown in Fig. 15.

Moreover, the critical angle decreases as the value of $V_{p d \sigma}$ increases. This behavior seems as if the large $d-d$ transfer would not favor the FM2 state. With increasing the $V_{p d \sigma}$ value, the energy difference between the $t_{2 g}$ and $e_{g}$ levels (i.e., $\Delta_{e_{g}}$ ) are increased, which is approximately given in as Eq. (33). This increase of $\Delta_{e_{g}}$ strongly suppresses the characteristic perturbational energy gain for the FM2 spin configuration $t_{3}^{2} j /\left(u^{\prime}+\Delta_{e_{g}}\right)^{2}$. Consequently, the AFM phase tends to be enhanced while the FM phase suppressed with a large $V_{p d \sigma}$ value.

\section{§4. Summary, Conclusions and Discussions}

In this paper, we have studied the magnetic and orbital ordered states and their phase transitions in perovskitetype Ti oxides by utilizing the effective spin and pseudospin Hamiltonian. The Hamiltonian is derived by the second-order perturbational expansion excluding the doubly occupancy of the $t_{2 g}$ states in the limit of the strong Coulomb repulsion. The full degeneracies of $\mathrm{Ti}$ $3 d$ and $\mathrm{O} 2 p$ orbitals and $d$ - $d$ Coulomb and exchange in- 
teractions are taken into account. Moreover, the effects of the $\mathrm{GdFeO}_{3}$-type distortion and the $d$-type JT distortion are also considered through the anisotropic transfer amplitudes and the Ti $3 d$ level splitting. We neglect the spin-orbit interaction in the region of large JT distortion. According to the mean-field calculations, in the $d$-type JT distortion, the $(y z, z x, y z, z x)$-type orbital order is strongly favored by the $\mathrm{GdFeO}_{3}$-type distortion due to the large amplitudes of transfers between neighboring $e_{g}$ and $t_{2 g}$ orbitals. The $\operatorname{AFM}(\mathrm{A})$ phase with this orbital ordering is stabilized in the moderately distorted region. This $\operatorname{AFM}(\mathrm{A})$ solution is obtained theoretically for the first time in the perovskite-type Ti oxides as far as we know. This phase may be detected by the neutronscattering experiments.

With decreasing the Ti-O-Ti bond angle, the $\operatorname{AFM}(\mathrm{A})$-to-FM phase transition arises. The $e_{g}$-orbital degrees of freedom play an important role on this magnetic phase transition. The energy difference between $\operatorname{AFM}(\mathrm{A})$ and FM solutions is consistent with the value expected from experimentally observed $T_{N}$ and $T_{C}$.

The $\mathrm{GdFeO}_{3}$-type distortion makes the $t_{2 g}$ orbitals hybridized with the $e_{g}$ orbitals. In the $d$-type JT distortion, $(y z, z x, y z, z x)$-type orbital state is stabilized due to the large amplitudes of the transfers between neighboring $t_{2 g}$ and $e_{g}$ orbitals. Through the AFM(A)-to-FM phase transition, this orbital state hardly changes. Since the orbital state changes negligibly through the magnetic phase transition, the spin-exchange interaction along the $c$-axis which is characterized by the second-order perturbational energy gains changes from positive (AFM spin coupling) to negative (FM spin coupling) nearly continuously and is suppressed to almost zero at the phase boundary. On the contrary, the strong FM spin coupling is constantly realized in the $a b$-plane. Consequently, the strong two-dimensionality in the spin coupling is realized near the phase boundary. This fact is a possible reason for the critical suppression of $T_{N}$ and $T_{C}$ near the phase boundary. We expect strong quantum fluctuations at the phase boundary. A slight change in the orbital state occurs at the phase boundary, and it causes a very weak first-order phase transition in this system.

This two-dimensional character in the spin coupling may be reflected on the dispersion relations of the spin waves which can be obtained by neutron-scattering experiments. When the spin coupling in this system is mapped on the Heisenberg model given in Eq. (38), the dispersion relation of the spin wave in the $\operatorname{AFM}(\mathrm{A})$ state $\left(J_{\text {Heis }}^{a, b}<0, J_{\text {Heis }}^{c}>0\right)$ is given by

$$
\begin{aligned}
& \varepsilon(\boldsymbol{k})= \\
& \sqrt{\left[-J_{\text {Heis }}^{a, b}\left\{2-\left(\cos k_{x} a+\cos k_{y} a\right)\right\}+J_{\text {Heis }}^{c}\right]^{2-}\left(J_{\text {Heis }}^{c} \cos k_{z} a\right)^{2}},
\end{aligned}
$$

where $a$ is a lattice constant. This expression can be easily obtained by the conventional Holstein-Primakoff transformation. We can rewrite this dispersion relation near the $\left(k_{x}, k_{y}, k_{z}\right)=(0,0,0)$ point as,

$$
\varepsilon(\boldsymbol{k})=\sqrt{-J_{\text {Heis }}^{a, b} J_{\text {Heis }}^{c}\left(k_{x}^{2} a^{2}+k_{y}^{2} a^{2}\right)+J_{\text {Heis }}^{c} k_{z}^{2} a^{2}} .
$$

If $\left|J_{\text {Heis }}^{c}\right| \ll\left|J_{\text {Heis }}^{a, b}\right|$ is realized, the ratio for the slope of the dispersion in the $z$-direction to that in the $x, y$ directions (i.e., $\sqrt{\left|J_{\text {Heis }}^{c} / J_{\text {Heis }}^{a, b} J_{\text {Heis }}^{c}\right|}=\sqrt{\left|J_{\text {Heis }}^{c} / J_{\text {Heis }}^{a, b}\right|}$ will be suppressed strongly. In this way, the strong two-dimensionality in the spin coupling near the phase boundary which is predicted in our theory will be reflected on the dispersion. Besides, we can expect to observe experimentally:

- in AFM and FM phases near the phase boundary, or in $\mathrm{SmTiO}_{3}$ and $\mathrm{GdTiO}_{3}$, the orbital states are similar to that in $\mathrm{YTiO}_{3}$.

- AFM state near the phase bounadary is A-type.

- Strong quantum fluctuations due to the two dimensionality exist near the phase boundary.

Moreover,we can control the dimensionality by $R$-site substitution in this system.

Similar effective spin and pseudospin Hamiltonians have also been proposed for perovskite-type Mn oxides in which not only diagonal transfers but also off-diagonal transfers between neighboring $e_{g}$ orbitals are taken into account ${ }^{1-3,29-32)}$. In those Hamiltonians, the transfer integrals reflect the realistic cubic perovskite-type structure. However, the effects of the $\mathrm{GdFeO}_{3}$-type distortion are not introduced in those models. It should be noted that the hybridization between $t_{2 g}$ and $e_{g}$ orbitals driven by the $\mathrm{GdFeO}_{3}$-type distortion is of crucial importance for the stabilizing the $\operatorname{AFM}(\mathrm{A})$ and FM2 phases in perovskite-type Ti oxides, as we have discussed.

In connection to the double exchange mechanism, we point out the possibility of an interesting large negative magnetoresistance around the $\operatorname{AFM}(\mathrm{A})$-to-FM2 transition point when carriers are doped by substitution of the $R$-site with an element with different valence such as Sr. Since the $c$-axis conduction is strongly favored by the ferromagnetic ordering, the applied magnetic field in the $\operatorname{AFM}(\mathrm{A})$ phase near the transition point may contribute to drastically reduce the resistivity and favors the appearance of a metal under the carrier doping.

\section{Acknowledgement}

M. M. thanks T. Mizokawa and H. Asakawa for valuable discussions and useful comments. This work is supported by "Research for the Future Program" (JSPSRFTF97P01103) from the Japan Society for the Promotion of Science.

1) K. I. Kugel and D. I. Khomskii, Pisma. Zh. Eksp. Teor. Fiz. 15 (1972) 629.

2) K. I. Kugel and D. I. Khomskii, Zh. Eksp. Teor. Fiz. 64 (1973) 1429. [Sov. Phys. JETP. 37, (1973) 725.]

3) K. I. Kugel and D. I. Khomskii, Sov. Phys. Usp 25 (1982) 231

4) D. I. Khomskii and K. I. Kugel, Solid State Commun. 13 (1973) 763.

5) For a review see M. Imada, A. Fujimori and Y. Tokura: Rev. Mod. Phys. 70 (1998) 1039.

6) D. A. Maclean, H.-N. Ng and J. E. Greedan, J. Solid State Chem. 30 (1979) 35

7) J. Kanamori, J. Phys. Chem. Solids, 10 (1959) 87.

8) J. Kanamori, J. Appl. Phys. Suppl. 31 (1960) 145.

9) J. B. Goodenough, Phys. Rev. 100, (1955) 564.

10) J. B. Goodenough, Magnetism and the Chemical Bond, (N Y. -London, 1963).

11) J. Akimitsu et al.: unpublished. 
12) J. P. Goral, J. E. Greedan and D. A. Maclean, J. Solid State Chem. 43 (1982) 244.

13) J. E. Greedan; J. Less-Common Met. 111 (1985) 335.

14) Y. Okimoto, T. Katsufuji, Y. Okada, T. Arima and Y. Tokura, Phys. Rev. B 51 (1995) 9581.

15) T. Katsufuji, Y. Taguchi and Y. Tokura, Phys. Rev. B 56 (1997) 10145.

16) J. P. Goral and J. E. Greedan, J. Magn. Mater. 37 (1983) 315 .

17) J. D. Garret and J. E. Greedan, Inorg. Chem. 20 (1981) 1025 .

18) T. Mizokawa and A. Fujimori, Phys. Rev. B 54 (1996) 5368.

19) T. Mizokawa and A. Fujimori, Phys. Rev. B 51 (1995) 12 880 .

20) H. Sawada, N. Hamada and K. Terakura, Physica B 46 (1997) 237-238.

21) M. Mochizuki and M. Imada, J. Phys. Soc. Jpn. 69 (2000) 1982.

22) B. H. Brandow, Adv. Phys. 26 (1977) 651.

23) J. Kanamori, Prog. Theor. Phys. 30 (1963) 275.

24) W. A. Harrison, Electronic Structure and the Properties of Solids (Dover, New York, 1989).

25) T. Saitoh, A. E. Bocquet, T. Mizokawa and A. Fujimori, Phys. Rev. B 52 (1995) 7934.

26) A. E. Bocquet, T. Mizokawa, K. Morikawa, A. Fujimori, S. R. Barman, K. Mati, D. D. Sarma, Y. Tokura and M. Onoda, Phys. Rev. B 53 (1996) 1161.

27) J. C. Slater and G. F. Koster, Phys. Rev. 94 (1954) 1498.

28) M. D. Mermin and H. Wagner, Phys. Rev. Lett. 17 (1966) 1133.

29) C. Castellani, C. R. Natoli and J. Ranninger, Phys. Rev. B 18 (1978) 4945.

30) W. Koshibae, Y. Kawamura, S. Ishihara, S. Okamoto, J. Inoue and S. Maekawa, J. Phys. Soc. Jpn. 66 (1997) 957.

31) S. Ishihara, J. Inoue and S. Maekawa, Physica C 263 (1996) 130 .

32) S. Ishihara, J. Inoue and S. Maekawa, Phys. Rev. B 55 (1997) 8280 . 